\title{
Logics for Propositional Determinacy and Independence
}

\author{
Valentin Goranko \\ Department of Philosophy \\ Stockholm University \\ Sweden \\ Department of Mathematics \\ University of Johannesburg \\ South Africa*
}

\author{
Antti Kuusisto \\ FB3: Mathematics/Computer Science \\ University of Bremen \\ Germany
}

\begin{abstract}
This paper investigates formal logics for reasoning about determinacy and independence. Propositional Dependence Logic $\mathcal{D}$ and Propositional Independence Logic $\mathcal{I}$ are recently developed logical systems, based on team semantics, that provide a framework for such reasoning tasks. We introduce two new logics $\mathcal{L}_{\mathrm{D}}$ and $\mathcal{L}_{1}$, based on Kripke semantics, and propose them as alternatives for $\mathcal{D}$ and $\mathcal{I}$, respectively. We analyse the relative expressive powers of these four logics and discuss the way these systems relate to natural language. We argue that $\mathcal{L}_{\mathrm{D}}$ and $\mathcal{L}_{1}$ naturally resolve a range of interpretational problems that arise in $\mathcal{D}$ and $\mathcal{I}$. We also obtain sound and complete axiomatizations for $\mathcal{L}_{\mathrm{D}}$ and $\mathcal{L}_{\mathrm{I}}$.
\end{abstract}

\section{Introduction}

In this paper we investigate the notions of propositional determinacy and propositional independence. We begin with a brief overview of related concepts.

\subsection{Dependence and independence: brief historical notes}

Dependence and independence are abstract notions that have played an important role in mathematics and the natural sciences since antiquity. Today the concepts are omnipresent in virtually all fields of science. There exists a wide range of different scientific notions of dependence, e.g., statistical correlation, the causal relationship, and functional dependence. Likewise, the notion of independence has different meanings in different contexts, e.g., probabilistic and linear independence in mathematics, as well as political and behavioural independence in social vernacular.

An early formal logical analysis of dependence was proposed in Gre39]1. Dependencies in relational databases have been studied since (at least) the pioneering

\footnotetext{
${ }^{*}$ Visiting professorship

${ }^{1}$ Also available as a reprint in SvE88.
} 
work of Codd in the early 1970's. Of the many relevant references we only note here Armstrong's work ([Arm74]) which provides a set of axioms for the notion of functional dependence in databases.

The concept of dependence has also appeared in various philosophical contexts under various different names. From the point of view of the current article, the notion of supervenience is perhaps the most important related concept. The paper MB14 describes supervenience formally as follows:

"A set of properties $A$ supervenes upon another set $B$ just in case no two things can differ with respect to A-properties without also differing with respect to their $B$ properties. In slogan form, 'there cannot be an A-difference without a B-difference'."

On the formal level, perhaps the closest in spirit to the present study regarding dependence is Humberstone's logical formalisation and study of supervenience as a generalisation of logical consequence in, e.g., Hum92, Hum93, Hum98. Another notion related to the current paper is the notion of contingency. Formal investigations of contingency can be found in, inter alia, [MR66, Hum95, Hum02, as well as Piz07, Piz13]. For a recent study of the contingency operator in various modal logics see FWvD15.

We also mention two very recent and closely related papers written after the appearance of the earlier version [GK16] of the present paper. The two papers have at least partially been written as a response to GK16]. The first one of them is [Fan16] which develops a formal modal logic of supervenience and also addresses some research questions raised in [GK16] and reiterated here in Section 8. The other one is [Hum17] which explores, inter alia, connections between supervenience and dependence and discusses in detail some aspects of [GK16].

In the context of logical semantics, the notion of independence has been investigated perhaps most prominently in Independence Friendly (IF) Logic originally defined in [HS89]; see also [Hin96]. IF logic was first formulated in terms of gametheoretic semantics, and no compositional semantics for that logic was originally available. Later on, Hodges developed ([Hod97]) a compositional semantics for the system, currently know as team semantics. The idea of team semantics, in turn, lead to Väänänen's development of Dependence Logic in Vää07]. Dependence logic sparked a renewed interest in logical formalisation and analysis of dependence and initiated an active related research programme. For an overview of the work in that direction, see [Kon13, GV14] and the references therein.

\subsection{Propositional logics of dependence and independence based on team semantics}

Väänänen's Dependence Logic extends classical first-order logic with dependence atoms

$$
\mathrm{D}\left(x_{1}, \ldots, x_{k} ; y\right)
$$

with the intuitive meaning that the choice of an interpretation for $y$ is functionally determined by the choices of interpretations for $x_{1}, \ldots, x_{k}$ in evaluation games based on game-theoretic semantics. Since the introduction of dependence atoms, research 
on logics based on team semantics has flourished and several kinds of related logical systems have been investigated.

A propositional modal variant of Dependence Logic, called Modal Dependence Logic, was defined in [Vää08. That logic extends the syntax of ordinary modal logic with a new operator $\mathrm{D}$ and formulae $\mathrm{D}\left(p_{1}, \ldots, p_{k} ; q\right)$ with the intuitive interpretation that the truth values of $p_{1}, \ldots, p_{k}$ determine the truth value of $q$. The propositional fragment of Modal Dependence Logic extended with D gives rise to Propositional Dependence Logic $\mathcal{D}$. In the logic $\mathcal{D}$, sets of propositional assignments are called teams; recall that a propositional assignment is simply a function from a set of atomic proposition symbols to the Boolean domain $\{0,1\}$. Intuitively, a team can be regarded as a set of possible worlds. A formula $\mathrm{D}(p ; q)$ is then defined to be true in a team $W$ if and only if each pair of possible worlds $w, u \in W$ that give the same truth value to $p$, must also give the same value to $q$. The more complex atoms $\mathrm{D}\left(p_{1}, \ldots, p_{k} ; q\right)$ are interpreted in a similar fashion: any pair of worlds in $W$ that agree on the truth values of $p_{1}, \ldots, p_{k}$, must also agree on the value of $q$ (see Section 2 for the formal definition.) The propositions $p_{1}, \ldots, p_{k}$ are said to determine $q$.

The notion of independence which we investigate in this paper originates from Independence Logic defined in GV13. Analogously to Dependence Logic, Independence Logic extends first-order logic by independence atoms

$$
\left(x_{1}, \ldots, x_{i}\right) \mathrm{I}_{\left(y_{1}, \ldots, y_{j}\right)}\left(z_{1}, \ldots, z_{k}\right),
$$

with the intuitive meaning that for any fixed set of values for $y_{1}, \ldots, y_{j}$, the possible values for $x_{1}, \ldots, x_{i}$ are independent of the possible values for $z_{1}, \ldots, z_{k}$. A propositional variant of Independence Logic, called Propositional Independence Logic (and denoted here by $\mathcal{I}$ ) has been investigated in the literature (in, e.g., KMSV14, Yan14]), and it relates to Independence Logic the same way Propositional Dependence Logic relates to Dependence Logic.

Dependence Logic and Independence Logic, together with the Inclusion Logic of Gal12], are currently the central logical systems studied in the framework of team semantics. It is also worth noting here that Propositional Dependence Logic is closely related to inquisitive logic [Cia09, CR11. In particular, the system InqL of inquisitive logic is a propositional team-based logic equi-expressive with $\mathcal{D}$. For investigations on the relations between inquisitive logic and $\mathcal{D}$, see, e.g., Yan14, YV16, Cia16a, Cia16b.

\subsection{The content and contributions of this paper}

While many of the semantic choices underlying Propositional Dependence Logic $\mathcal{D}$ are natural and justified, we will identify in this paper a range of issues that are problematic. One such issue is the interpretation of formulae that use combinations of determinacy operators $\mathrm{D}$ and disjunctions $\vee$. We will argue that while team semantics gives a sensible interpretation to formulae $\mathrm{D}\left(p_{1}, \ldots, p_{k} ; q\right)$ as well as disjunctive formulae $\varphi \vee \psi$ free of operators $\mathrm{D}$, interpretations of certain simple formulae that combine $\mathrm{D}$ and $\vee$ become strange from the point of view of natural language. We 
also discuss similar issues related to the team semantics interpretation of negation $\neg$ (together with $\mathrm{D})$.

Motivated by the interpretational problems of $\mathcal{D}$, we develop here an alternative natural logic of determinacy called Propositional Logic of Determinacy and denoted by $\mathcal{L}_{\mathrm{D}}$. The logics $\mathcal{D}$ and $\mathcal{L}_{\mathrm{D}}$ have essentially the same set of formulae 21 but the semantic approaches differ. Instead of team semantics, the system $\mathcal{L}_{\mathrm{D}}$ is essentially based on Kripke semantics. A formula $\mathrm{D}\left(\varphi_{1}, \ldots, \varphi_{k} ; \psi\right)$ is true 3 in a possible world $w$ if the set $R(w)$ of accessible alternatives of $w$ satisfies the determinacy condition: for all $u, v \in R(w)$, if $u$ and $v$ agree on the truth values of each $\varphi_{i}$, they also agree on the truth value of $\psi$. The Boolean connectives as well as proposition symbols are interpreted in $\mathcal{L}_{\mathrm{D}}$ in the same way as in Kripke semantics, and thus $\mathcal{L}_{\mathrm{D}}$ can be regarded as a modal logic with a generalized modality $\mathrm{D}$ that talks about determinacy rather than possibility or necessity. Mainly in order to keep matters technically simple in this initial work on $\mathcal{L}_{\mathrm{D}}$, we assume the accessibility relation $R$ to be the universal relation, so the set of successors of any world $w$ is in fact the whole domain of the model. At the end of the paper we briefly discuss the general case with other kinds of accessibility relations.

As an important part of our discourse on $\mathcal{L}_{\mathrm{D}}$, we present a range or arguments for the naturalness of $\mathcal{L}_{\mathrm{D}}$ in relation to natural language. In particular, we argue that $\mathcal{L}_{\mathrm{D}}$ resolves reasonably well the interpretational problems that we identify for $\mathcal{D}$.

It turns out that Propositional Independence Logic $\mathcal{I}$ is burdened by virtually the same issues as Propositional Dependence Logic $\mathcal{D}$, and these issues can be remedied by defining Propositional Logic of Independence $\mathcal{L}_{\mathrm{l}}$ analogously to $\mathcal{L}_{\mathrm{D}}$ but based on the independence operator I rather than the dependence operator $\mathrm{D}$.

In addition to introducing the logics $\mathcal{L}_{\mathrm{D}}$ and $\mathcal{L}_{\mathrm{I}}$ and discussing how they, as well as $\mathcal{D}$ and $\mathcal{I}$, relate to each other and to natural language, we also provide a comparative analysis of the expressive powers of these four logics. We show that, while $\mathcal{D}$ and $\mathcal{I}$ are both strictly contained in $\mathcal{L}_{\mathrm{D}}$ and $\mathcal{L}_{\mathrm{l}}$, the latter two logics are equally expressive. In fact, we establish in Sections 3 and 4 that $\mathcal{L}_{\mathrm{D}}$ and $\mathcal{L}_{\mathrm{I}}$ are maximally expressive, or expressively complete, in a certain natural sense. Since it is well known from the literature on team semantics that $\mathcal{D}$ is strictly contained in $\mathcal{I}$, we eventually obtain a complete classification of the relative expressive powers of the four logics.

We also prove that while both $\mathcal{D}$ and $\mathcal{I}$ translate into both $\mathcal{L}_{\mathrm{D}}$ and $\mathcal{L}_{\mathrm{l}}$, there exists no compositional translation 4 from either of the team-semantics-based logics into $\mathcal{L}_{\mathrm{D}}$ or $\mathcal{L}_{1}$. Intuitively, this indicates that team semantics and the Kripke-style semantics of $\mathcal{L}_{\mathrm{D}}$ and $\mathcal{L}_{\mathrm{I}}$ are substantially different logical frameworks, at least from the technical point of view.

\footnotetext{
${ }^{2}$ Strictly speaking, $\mathcal{L}_{\mathrm{D}}$ has more formulae than $\mathcal{D}$ because of the typical syntactic restrictions applied in $\mathcal{D}$ and team semantics in general. This issue is discussed in more detail in the sections below.

${ }^{3}$ This truth definition was first suggested as an alternative to team semantics in Kuu14 and its later versions such as, e.g., Kuu15.

${ }^{4}$ See Section 6.4 for the definition of compositional translations.
} 
In addition to studying expressivity issues, we provide sound and complete axiomatizations for $\mathcal{L}_{\mathrm{D}}$ and $\mathcal{L}_{1}$. This turns out to be a relatively straightforward, yet interesting exercise, due to certain close connections - to be identified belowbetween $\mathcal{L}_{\mathrm{D}}, \mathcal{L}_{\mathrm{I}}$ and Contingency Logic from [MR66]. Contingency Logic is the variant of modal logic with a modality $\mathrm{C}$, where $\mathrm{C} \varphi$ is interpreted to mean that $\varphi$ is non-contingent at the state of evaluation, i.e., has the same truth value at every successor of that state. We also show that there do not exist finite axiomatisations for $\mathcal{L}_{\mathrm{D}}$ and $\mathcal{L}_{\mathrm{l}}$, assuming a standard notion of an axiomatic deduction system.

The structure of this paper is as follows. In Section 2 we provide the necessary background for the rest of the paper, including definitions of the logics $\mathcal{D}$ and $\mathcal{I}$ based on team semantics. In Sections 3 and 4 we define the logics $\mathcal{L}_{\mathrm{D}}$ and $\mathcal{L}_{\mathrm{I}}$ and study their basic properties. Section [5 analyses the logics $\mathcal{D}, \mathcal{I}, \mathcal{L}_{\mathrm{D}}$ and $\mathcal{L}_{\mathrm{I}}$ in relation to natural language. Section 6 investigates expressivity issues and Section 7 provides sound and complete axiomatizations for $\mathcal{L}_{\mathrm{D}}$ and $\mathcal{L}_{\mathrm{l}}$. Section 8 briefly discusses a range of future research directions and concludes the paper.

The agenda and main idea of the current paper, i.e., investigating dependence logic with a Kripke-style semantics instead of team semantics, has been first mentioned and briefly motivated in [Kuu14 and its later incarnations such as, e.g., Kuu15. Similar ideas have subsequently been developed in Cia16b]. The current paper develops the ideas of [Kuu14] in detail, and furthermore, provides an extensive collection of related technical results concerning the expressivity and axiomatizability of $\mathcal{L}_{\mathrm{D}}$ and related systems.

Section 6.7 .2 of Cia16b contains an explicit comparison of $\mathcal{L}_{\mathrm{D}}$ with a logic $\mathrm{InqB}^{\Rightarrow}$ that employs a Kripke-style approach (with some extra machinery) to semantics and thereby exhibits the same principle as $\mathcal{L}_{\mathrm{D}}$ that dependence statements are modal statements. One of the main differences between InqB $\Rightarrow$ and $\mathcal{L}_{\mathrm{D}}$ is that $\mathrm{InqB}^{\Rightarrow}$ contains an explicit machinery for questions and thereby allows semantically elaborate assertions about, e.g., dependence. Dependence statements in $\mathcal{L}_{\mathrm{D}}$ are relations between statements, while in $\mathrm{InqB}^{\Rightarrow}$, dependence statements are construed

as a relation between questions. See Sections 6.7.2 and 6.5 of Cia16b for further details.

\section{Preliminaries and background}

\subsection{Functional determinacy}

Determinacy of a function by a set of functions is a central concept in this article. We will define it here in the general setting, though will use it further only on Boolean functions.

Definition 2.1. Let $k \in \mathbb{Z}_{+}$be a positive integer and let $X, X_{1}, \ldots, X_{k}, U$ be nonempty sets. Let $f: U \rightarrow X$ be function, and consider a family of functions

$$
\left\{f_{i}: U \rightarrow X_{i} \mid i=1, \ldots, k\right\} .
$$

Given a set $W \subseteq U$, we say that the function $f$ is determined by the family of functions $\left\{f_{1}, \ldots, f_{k}\right\}$ on $W$, or that the family $\left\{f_{1}, \ldots, f_{k}\right\}$ determines the function $f$ 
on $W$, if there exists a function $F: X_{1} \times \ldots \times X_{k} \rightarrow X$ such that $f$ is the composition of $F$ and the functions $f_{1}, \ldots, f_{k}$ on $W$, that is, $f(w)=F\left(f_{1}(w), \ldots, f_{k}(w)\right)$ for every $w \in W$. We also fix this definition in the following special case: we say that a function $g: U \rightarrow X$ is determined by $\emptyset$ on $W \subseteq U$ if $g$ is constant on $W$, i.e., $g\left(w_{1}\right)=g\left(w_{2}\right)$ for all $w_{1}, w_{2} \in W$.

This definition generalises straightforwardly to determinacy of a function by any family of functions $\left\{f_{i}: U \rightarrow X_{i} \mid i \in I\right\}$ indexed with an arbitrary (possibly infinite) set $I$.

Equivalently, $f$ is determined by the (possibly empty) family $\left\{f_{1}, \ldots, f_{k}\right\}$ on $W$ if and only if the following condition holds.

Det: For every $w_{1}, w_{2} \in W$, it holds that if $f_{i}\left(w_{1}\right)=f_{i}\left(w_{2}\right)$ for each $i=1, \ldots, k$, then $f\left(w_{1}\right)=f\left(w_{2}\right)$.

Indeed, if $f$ is the composition of a mapping $F$ with $f_{1}, \ldots, f_{k}$ on $W$ and $f_{i}\left(w_{1}\right)=$ $f_{i}\left(w_{2}\right)$ for each $i=1, \ldots, k$, then $F\left(f_{1}\left(w_{1}\right), \ldots, f_{k}\left(w_{1}\right)\right)=F\left(f_{1}\left(w_{2}\right), \ldots, f_{k}\left(w_{2}\right)\right)$, i.e., $f\left(w_{1}\right)=f\left(w_{2}\right)$. Conversely, if the condition Det holds, then we can define a mapping $F: X_{1} \times \ldots \times X_{k} \rightarrow X$ as follows:

$$
F\left(x_{1}, \ldots, x_{k}\right)= \begin{cases}f(w) & \text { if } x_{1}=f_{1}(w), \ldots, x_{k}=f_{k}(w), \text { for some } w \in W \\ x & \text { otherwise (where } x \in X \text { is arbitrarily fixed) }\end{cases}
$$

The condition Det guarantees that this is well-defined.

We let $\operatorname{Det}_{W}\left(f_{1}, \ldots, f_{k} ; f\right)$ denote the assertion that $f$ is determined by the family of functions $\left\{f_{1}, \ldots, f_{k}\right\}$ on $W$. When $k=0$, we write $\operatorname{Det}_{W}(\emptyset ; f)$.

\subsection{Preliminaries concerning propositional logic}

We typically denote formulae by $\varphi, \psi, \chi, \theta, \alpha, \beta$ and sets of formulae by $\Phi, \Psi$. Throughout the paper, we let $P R O P$ denote a fixed countably infinite set of proposition symbols. All formulae considered in the paper will be assumed to be built over PROP.

Let $\Phi=\left\{\varphi_{1}, \ldots, \varphi_{k}\right\}$ be a finite nonempty set of formulae. We define a set $\operatorname{DNF}(\Phi)$ as follows.

1. For each subset $S \subseteq\{1, \ldots, k\}$, let $\psi_{S}$ denote the conjunction $\psi_{1} \wedge \ldots \wedge \psi_{k}$ such that

$$
\psi_{i}= \begin{cases}\varphi_{i} & \text { if } i \in S \\ \neg \varphi_{i} & \text { if } i \notin S\end{cases}
$$

2. Let $\operatorname{Conj}(\Phi):=\left\{\psi_{S} \mid S \subseteq\{1, \ldots, k\}\right\}$. The formulae in $\operatorname{Conj}(\Phi)$ are called types over $\Phi$.

3. Define $\operatorname{DNF}(\Phi):=\{\bigvee U \mid U \subseteq \operatorname{Conj}(\Phi)\}$

We call the formulae in $D N F(\Phi)$ type normal form formulae over $\Phi$. 
In the above definition, $\bigvee \emptyset$ is assumed to be the formula $p \wedge \neg p$ for some proposition symbol $p \in P R O P$. We will not assume that the logical constant symbols $\top, \perp$ are available as primitives in the languages we consider. However, we will use these symbols as abbreviations for the formulae $p \vee \neg p$ and $p \wedge \neg p$, respectively. For technical convenience, we define $\operatorname{DNF}(\emptyset):=\{\top, \perp\}$ and $\operatorname{Conj}(\emptyset):=\{\top\}$, and thus we let $T$ be the unique type over $\emptyset$.

When we write a formula $\theta\left(q_{1}, \ldots, q_{k}\right)$, we indicate that all the propositional variables occurring in $\theta$ are amongst $q_{1}, \ldots, q_{k}$. Given a formula $\theta=\theta\left(q_{1}, \ldots, q_{k}\right)$ and a tuple of formulae $\varphi_{1}, \ldots, \varphi_{k}$, we denote by $\theta\left(\varphi_{1}, \ldots, \varphi_{k}\right)$ the result of a uniform substitution of $\varphi_{1}, \ldots, \varphi_{k}$, respectively, for $q_{1}, \ldots, q_{k}$ in the formula $\theta$.

Definition 2.2. Let $L$ be a logic. A relation of equivalence $\equiv_{L}$ between formulae of $L$ satisfies the equivalent replacements property (ER) (with respect to $\equiv_{L}$ ), if for every $L$-formula $\theta\left(q_{1}, \ldots, q_{k}\right)$ and all tuples of $\varphi_{1}, \ldots, \varphi_{k}, \psi_{1}, \ldots, \psi_{k}$ such that $\varphi_{i} \equiv_{L} \psi_{i}$ for each $i=1, \ldots, k$, it holds that $\theta\left(\varphi_{1}, \ldots, \varphi_{k}\right) \equiv_{L} \theta\left(\psi_{1}, \ldots, \psi_{k}\right)$.

\subsection{State description models}

Recall that $P R O P$ denotes a fixed countably infinite set of proposition symbols. An assignment for PROP is any mapping $f: P R O P \rightarrow\{0,1\}$. Following Carnap, we occasionally call assignments also state descriptions.

Any (possibly empty) set $W$ of state descriptions will be called a state description model (or $S D$-model). The reason we include the empty model in the picture is technical and related to the the fact that we will deal, inter alia, with logics based on team semantics. In team semantics, as we will see, the empty team plays an important role.

Especially in the more technical parts of the paper, we often talk about points or worlds of $W$ rather than assignments or state descriptions.

\subsection{Universal modality}

In this paper we use a variant $\mathcal{L}_{\mathcal{U}}$ of the modal logic with the universal modality from GP92. Formally, the syntax of the logic $\mathcal{L}_{\mathcal{U}}$ is given by the grammar

$$
\varphi::=p|\neg \varphi|(\varphi \rightarrow \varphi) \mid[\mathrm{U}] \varphi
$$

where $p \in P R O P$. We define $\langle\mathrm{U}\rangle$ to be the dual of $[\mathrm{U}]$, i.e., $\langle\mathrm{U}\rangle \varphi:=\neg[\mathrm{U}] \neg \varphi$.

The semantics of $\mathcal{L}_{\mathcal{U}}$ is defined with respect to SD-models $W$ and assignments $w \in W$ as follows.

$$
\begin{array}{lll}
W, w \models_{\mathcal{U}} p & \text { iff } & w(p)=1 \\
W, w \models_{\mathcal{U}} \neg \varphi & \text { iff } & W, w \models_{\mathcal{U}} \varphi \\
W, w \models_{\mathcal{U}} \varphi \rightarrow \psi & \text { iff } & W, w \models_{\mathcal{U}} \varphi \text { or } W, w \models_{\mathcal{U}} \psi \\
W, w \models_{\mathcal{U}}[\mathrm{U}] \varphi & \text { iff } & W, u \models_{\mathcal{U}} \varphi \text { for all } u \in W
\end{array}
$$

Thus, $W, w \models_{\mathcal{U}}\langle\mathrm{U}\rangle \varphi$ iff $W, u \models_{\mathcal{U}} \varphi$ for some $u \in W$.

As customary in modal logic, we define $W \models_{\mathcal{U}} \varphi$ iff $W, w \models_{\mathcal{U}} \varphi$ for all $w \in W$. We also define, in the standard way, that $\models_{\mathcal{U}} \varphi$ iff $W \models_{\mathcal{U}} \varphi$ for all SD-models $W$. 


\subsection{Propositional Dependence Logic $\mathcal{D}$}

We now define Propositional Dependence Logic $\mathcal{D}$, which first appeared in the literature on team semantics as a syntactic fragment of Modal Dependence Logic, defined in [Vää08]. The paper [Vää08] did not make explicit references to $\mathcal{D}$, and the semantics for Modal Dependence Logic - including its propositional fragment-was formulated in [Vää08] in terms of Kripke models rather than SD-models.

Propositional Dependence Logic, with that explicit name, and variants of the logic have recently been studied in, e.g., HLSV14, HKMV15, Yan14, YV16]. The models for Propositional Dependence Logic are currently typically defined in the literature as SD-models where the set of proposition symbols in consideration is finite; thus the related SD-models are sets of finite state descriptions, i.e., finite assignments. For most purposes, it makes little difference whether SD-models with finite or infinite sets of proposition symbols are used. Similarly, it is mostly unimportant whether the models under consideration are Kripke models or SD-models. Such distinctions could, however, become more important in extensions and variants of the logics considered in this paper.

The syntax of Propositional Dependence Logic $\mathcal{D}$ is given by the following grammar (cf. [Vää08]),

$$
\varphi::=p|\neg p| \mathrm{D}\left(p_{1}, \ldots, p_{k} ; q\right)\left|\neg \mathrm{D}\left(p_{1}, \ldots, p_{k} ; q\right)\right|(\varphi \vee \varphi) \mid(\varphi \wedge \varphi)
$$

where $p, q, p_{1}, \ldots, p_{k} \in P R O P$ and $k \in \mathbb{N}$. When considering formulae $\mathrm{D}\left(p_{1}, \ldots, p_{k} ; q\right)$ where $k=0$, we writt $5 \varphi$ instead of $\mathrm{D}(; q)$ or $\mathrm{D}(\epsilon ; q)$, where $\epsilon$ denotes the empty sequence of proposition symbols.

We let $\operatorname{FOR}(\mathcal{D})$ denote the set of formulae of $\mathcal{D}$. Note that formulae of $\mathcal{D}$ are in negation normal form, and the operator $\mathrm{D}$ takes as inputs only proposition symbols.

Let $W$ be a state description model. The semantics of Propositional Dependence Logic $\mathcal{D}$, to be defined below, is based on team semantics 6 , given by the following clauses (cf. [Vää08, YV16]).

$$
\begin{array}{lll}
W \Vdash_{\mathcal{D}} p & \text { iff } & w(p)=1 \text { for all } w \in W \\
W \Vdash_{\mathcal{D}} \neg p & \text { iff } & w(p)=0 \text { for all } w \in W \\
W \Vdash_{\mathcal{D}} \mathrm{D}\left(p_{1}, \ldots, p_{k} ; q\right) & \text { iff } & \text { for all } u, v \in W, \text { if } u\left(p_{i}\right)=v\left(p_{i}\right) \\
& & \text { holds for all } i \leq k, \text { then } u(q)=v(q) \\
W \Vdash_{\mathcal{D}} \neg \mathrm{D}\left(p_{1}, \ldots, p_{k} ; q\right) & \text { iff } & W=\emptyset \\
W \Vdash_{\mathcal{D}} \varphi \wedge \psi & \text { iff } & W \Vdash_{\mathcal{D}} \varphi \text { and } W \Vdash_{\mathcal{D}} \psi \\
W \Vdash_{\mathcal{D}} \varphi \vee \psi & \text { iff } & U \Vdash_{\mathcal{D}} \varphi \text { and } V \Vdash_{\mathcal{D}} \psi \text { for some } U, V \subseteq W \\
& & \text { such that } U \cup V=W
\end{array}
$$

\footnotetext{
${ }^{5}$ Here C stands for 'constancy' but, as Humberstone has noted, it might be confused with 'contingency' which is, in fact, its opposite.

${ }^{6}$ Throughout this paper, the turnstile $\Vdash$ is reserved for logics based on team semantics and the turnstile $\models$ for logics with a Kripke-style semantics. The reader is warned that we often use different turnstiles when comparing different logics, and the related change of turnstile may sometimes be difficult to spot at first.
} 
We observe that $W \Vdash \mathrm{C} p$ iff $v(p)=u(p)$ for all $u, v \in W$, i.e., the truth value of $p$ is constant in $W$.

The rationale for the truth condition of $\neg \mathrm{D}\left(p_{1}, \ldots, p_{k} ; q\right)$, as stated in Vää07, p.24] and reiterated in [LV13, is as follows. Suppose we wish to maintain the same duality between $\mathrm{D}\left(p_{1}, \ldots, p_{k} ; q\right)$ and $\neg \mathrm{D}\left(p_{1}, \ldots, p_{k} ; q\right)$ as the one that holds for the truth conditions for $p$ and $\neg p$. We then end up with the definition that $W \Vdash_{\mathcal{D}} \neg \mathrm{D}\left(p_{1}, \ldots, p_{k} ; q\right)$ iff for all $u, v \in W$,

$$
u\left(p_{1}\right)=v\left(p_{1}\right) \wedge \ldots \wedge u\left(p_{k}\right)=v\left(p_{k}\right) \wedge u(q) \neq v(q),
$$

where the expression above is obtained by negating the condition provided in the truth definition of $\mathrm{D}\left(p_{1}, \ldots, p_{k} ; q\right)$ after the universal quantification of $u$ and $v 7$ We then observe that according to the obtained definition, $W \Vdash_{\mathcal{D}} \neg \mathrm{D}\left(p_{1}, \ldots, p_{k} ; q\right)$ iff $W=\emptyset$.

Another possible rationale for the truth definition of formulas $\neg \mathrm{D}\left(p_{1}, \ldots, p_{k} ; q\right)$ can be obtained via an algebraic interpretation of formulae, as given in [Roe13. Here formulae are associated with non-empty and downwards closed sets of SD-models. Such sets correspond to possible meanings of formulae. This approach leads to a Heyting algebra. Negation is interpreted as the pseudo-complement operation, and for the formula $\neg \mathrm{D}\left(p_{1}, \ldots, p_{n} ; q\right)$ this gives an interpretation that is equivalent to the clause given above, i.e., $\neg \mathrm{D}\left(p_{1}, \ldots, p_{n} ; q\right)$ is satisfied by an SD-model $W$ iff $W=\emptyset$. See Roe13 for further details.

While team semantics may seem strange at first, the following proposition justifies its naturalness with respect to propositional logic, i.e., the sublanguage of $\mathcal{D}$ without formulae of the type $\mathrm{D}\left(p_{1}, \ldots, p_{k} ; q\right)$ and $\neg \mathrm{D}\left(p_{1}, \ldots, p_{k} ; q\right)$. Recall that the turnstile $\models_{\mathcal{U}}$ refers to $\mathcal{L}_{\mathcal{U}}$.

Proposition 2.3. Let $\varphi$ be a formula of propositional logic in negation normal form. Then $W \Vdash_{\mathcal{D}} \varphi$ iff $W, w \models_{\mathcal{U}} \varphi$ for all $w \in W$. In other words, $W \Vdash_{\mathcal{D}} \varphi$ iff $W \models_{\mathcal{U}} \varphi$.

This proposition shows that team semantics simply lifts the semantics of propositional logic (in negation normal form) from the level of individual assignments onto the level of sets of assignments. Thus, team semantics can be used in scenarios where assertions (encoded by formulae of propositional logic) are made about sets of possible worlds, and the intention of the assertions is to claim that any world in the set satisfies the formula. We will consider examples of such scenarios in Section 5. where we discuss the relation between natural language and the logic $\mathcal{D}$.

\subsection{Propositional Independence Logic $\mathcal{I}$}

We next present Propositional Independence Logic $\mathcal{I}$ which was conceived as a fragment of Modal Independence Logic in [KMSV14] and studied further in, e.g.,

\footnotetext{
${ }^{7}$ In other words, if we abbreviate the truth condition for $p$ by $\forall w \in W: \Psi$ and the truth condition for $\mathrm{D}\left(p_{1}, \ldots, p_{k} ; q\right)$ by $\forall u, v \in W: \Psi^{\prime}$, then the truth condition for $\neg p$ is $\forall w \in W: \neg \Psi$ and thus we define the condition for $\neg \mathrm{D}\left(p_{1}, \ldots, p_{k} ; q\right)$ to be $\forall u, v \in W: \neg \Psi^{\prime}$. How natural this choice is exactly, is a question that calls for further analysis. We will briefly discuss issues related to this matter in Section 5]
} 
HKVV15]. Propositional Independence Logic relates to Independence Logic of GV13 in the same way $\mathcal{D}$ relates to Väänänen's Dependence Logic. There are, of course, different kinds of notions of (propositional) independence, and the logic $\mathcal{I}$ provides a formal approach to a particular such notion. The logic is similar in spirit to $\mathcal{D}$, being based on team semantics.

The syntax of $\mathcal{I}$ is given by the following grammar.

$$
\varphi::=p|\neg p|\left(p_{1}, \ldots, p_{k}\right) \mathrm{I}_{\left(r_{1}, \ldots, r_{m}\right)}\left(q_{1}, \ldots, q_{n}\right)|(\varphi \vee \varphi)|(\varphi \wedge \varphi)
$$

where $p$ and each of the symbols $p_{i}, r_{i}, q_{i}$ are proposition symbols in PROP. We denote by $F O R(\mathcal{I})$ the set of formulae of $\mathcal{I}$. The numbers $k$ and $n$ are positive integers and $m$ a non-negative integer. When $m=0$, the formula

$$
\left(p_{1}, \ldots, p_{k}\right) \mathrm{I}_{\left(r_{1}, \ldots, r_{m}\right)}\left(q_{1}, \ldots, q_{n}\right)
$$

is written $\left(p_{1}, \ldots, p_{k}\right) \mid\left(q_{1}, \ldots, q_{n}\right)$. Also, when any of the three tuples of proposition symbols in the formula $\left(p_{1}, \ldots, p_{k}\right) \mathrm{I}_{\left(r_{1}, \ldots, r_{m}\right)}\left(q_{1}, \ldots, q_{n}\right)$ contains exactly one formula, the brackets around the tuple are usually left out, as for example in the formula $p \mathrm{I}_{r} q$.

Notice that, in line with the definition of $\mathcal{I}$ in [KMSV14, HKVV15, negation and I can only be applied to propositional symbols. In particular, formulae

$$
\left(p_{1}, \ldots, p_{k}\right) \mathbf{I}_{\left(r_{1}, \ldots, r_{m}\right)}\left(q_{1}, \ldots, q_{n}\right)
$$

may not occur negated. The same convention applies to independence atoms in Independence Logic GV13.

The semantics of $\mathcal{I}$ is defined with respect to SD-models. Intuitively, the formula

$$
\left(p_{1}, \ldots, p_{k}\right) \mathbf{I}_{\left(r_{1}, \ldots, r_{m}\right)}\left(q_{1}, \ldots, q_{n}\right)
$$

asserts that when the truth values of the proposition symbols $r_{1}, \ldots, r_{m}$ are fixed, then the tuples of truth values of $\left(p_{1}, \ldots, p_{k}\right)$ and $\left(q_{1}, \ldots, q_{n}\right)$ are informationally independent in a way explained further after the formal truth definition of $\mathcal{I}$.

We use $\Vdash_{\mathcal{I}}$ as the semantic turnstile of $\mathcal{I}$. The formal semantic clauses of $\mathcal{I}$ for propositional literals and Boolean connectives are exactly the same as those for the logic $\mathcal{D}$, while the semantics of the formulae

$$
\left(p_{1}, \ldots, p_{k}\right) \mathbf{I}_{\left(r_{1}, \ldots, r_{m}\right)}\left(q_{1}, \ldots, q_{n}\right)
$$

is defined as follows. Let $W$ be a state description model. We define

$$
\left.W \Vdash_{\mathcal{I}}\left(p_{1}, \ldots, p_{k}\right)\right|_{\left(r_{1}, \ldots, r_{m}\right)}\left(q_{1}, \ldots, q_{n}\right)
$$

iff for all $w_{1}, w_{2} \in W$ that agree on $r_{1}, \ldots, r_{m}$ (i.e., are such that $w_{1}\left(r_{i}\right)=w_{2}\left(r_{i}\right)$ for each $i \in\{1, \ldots, m\})$, there exists some $v \in W$ such that

$$
\left(\bigwedge_{i \leq m} v\left(r_{i}\right)=w_{1}\left(r_{i}\right) \wedge \bigwedge_{i \leq k} v\left(p_{i}\right)=w_{1}\left(p_{i}\right) \wedge \bigwedge_{i \leq n} v\left(q_{i}\right)=w_{2}\left(q_{i}\right)\right)
$$


Thus, the formula $\left(p_{1}, \ldots, p_{k}\right) \mathrm{I}_{\left(r_{1}, \ldots, r_{m}\right)}\left(q_{1}, \ldots, q_{n}\right)$ asserts that for every tuple $\left(b_{1}, \ldots, b_{m}\right)$ of truth values for the propositions $r_{1}, \ldots, r_{m}$, if we restrict attention to the set $S$ of those assignments in $W$ that assign the values $b_{1}, \ldots, b_{m}$ to $r_{1}, \ldots, r_{m}$, then the following condition holds: the tuples of truth values for $\left(p_{1}, \ldots, p_{k}\right)$ and $\left(q_{1}, \ldots, q_{n}\right)$ are informationally independent of each other on $S$ in the sense that for every two assignments $w_{1}, w_{2} \in S$, there is an assignment $v \in S$ that combines $w_{1}$ restricted to $\left(p_{1}, \ldots, p_{k}\right)$ with $w_{2}$ restricted to $\left(q_{1}, \ldots, q_{n}\right)$.

It is worth noting that, intuitively, the formula $p \mathrm{I} q$ can be interpreted to state that nothing new can be concluded about the truth value of $p$ in a possible world $w$ by finding out the truth value of $q$ in $w$ (and vice versa): an agent who fully knows the model $W$ but has no idea which $w \in W$ is the actual world, cannot conclude anything new about the truth value of $q$ in the actual world by learning the truth value of $p$ in that world.

This interpretation explains the initially perhaps counterintuitive fact that the formula $p \mathrm{I} p$ is satisfiable even in nonempty models: $W \vdash_{\mathcal{I}} p \mathrm{I} p$ holds iff $p$ is constant in $W$, i.e., if every assignment in $W$ gives the same truth value to $p$. Indeed, $p$ being constant means exactly that nothing new can be concluded about the truth value $p$ in the actual world by learning the truth value of $p$ in the actual world. If $p$ was not constant, the truth value of $p$ in the actual world would obviously reveal new information.

It is worth pointing out here that in the semantics of $\mathcal{D}$ and $\mathcal{I}$, there is no explicit actual world present. Next we will consider the logics $\mathcal{L}_{\mathrm{D}}$ and $\mathcal{L}_{\mathrm{l}}$ whose semantics are given in a way similar to Kripke semantics in terms of pairs $(W, w)$, where $W$ is a SD-model and $w \in W$ an assignment which can be taken to correspond to an appointed actual world.

\section{Propositional logic of determinacy $\mathcal{L}_{\mathrm{D}}$}

We now introduce a new logic which extends propositional logic PL with dependence formulae $\mathrm{D}\left(\varphi_{1}, \ldots, \varphi_{k} ; \psi\right)$, where $\varphi_{1}, \ldots, \varphi_{k}, \psi$ are arbitrary formulae in the language. We call this logic Propositional Logic of Determinacy and denote it by $\mathcal{L}_{\mathrm{D}}$

Recall that $P R O P$ denotes a fixed countably infinite set of proposition symbols. The formulae of $\mathcal{L}_{\mathrm{D}}$ over PROP are defined by the following grammar.

$$
\varphi::=p|\neg \varphi|(\varphi \rightarrow \varphi) \mid \mathrm{D}(\varphi, \ldots, \varphi ; \varphi)
$$

where $p \in P R O P$, and where the tuple $(\varphi, \ldots, \varphi ; \varphi)$ contains $k+1$ formulae for any $k \in \mathbb{N}$. We consider the Boolean connectives $\wedge, \vee, \leftrightarrow$ as abreviations in the usual way. When $k=0$, we write $\mathrm{C} \varphi$ instead of $\mathrm{D}(\epsilon ; \varphi)$, where $\epsilon$ is the empty sequence of formulae. We let $\operatorname{FOR}\left(\mathcal{L}_{\mathrm{D}}\right)$ denote the set of formulae of $\mathcal{L}_{\mathrm{D}}$. Notice indeed that each of the operators $\neg, \rightarrow$, D can be freely used in the language of $\mathcal{L}_{\mathrm{D}}$; no syntactic restrictions apply.

Intuitively, $\mathrm{D}\left(\varphi_{1}, \ldots, \varphi_{k} ; \psi\right)$ means that the truth value of $\varphi$ is determined by the set of truth values of the formulae $\varphi_{1}, \ldots, \varphi_{k}$ on the SD-model in consideration. In particular, $\mathrm{C} \varphi$ means that the truth value of $\varphi$ is constant in the model. 
We define truth of an $\mathcal{L}_{\mathrm{D}}$-formula $\varphi$ at a state description $w$ in an SD-model $W$, denoted $\$, w \models_{\mathrm{D}} \varphi$, inductively on the structure of formulae as follows.

$$
\begin{array}{lll}
W, w \models_{\mathrm{D}} p & \text { iff } & w(p)=1 \\
W, w \models_{\mathrm{D}} \neg \varphi & \text { iff } & W, w \models_{\mathrm{D}} \varphi \\
W, w \models_{\mathrm{D}} \varphi \rightarrow \psi & \text { iff } & W, w \nvdash_{\mathrm{D}} \varphi \text { or } W, w \models_{\mathrm{D}} \psi \\
W, w \models_{\mathrm{D}} \mathrm{D}\left(\varphi_{1}, \ldots, \varphi_{k} ; \psi\right) & \text { iff } \quad & \text { for all } u, v \in W, \text { if the equivalence } \\
& & \left(W, u \models_{\mathrm{D}} \varphi_{i} \Leftrightarrow W, v \models_{\mathrm{D}} \varphi_{i}\right) \text { holds for } \\
& & \text { all } i \leq k, \text { then }\left(W, u \models_{\mathrm{D}} \psi \Leftrightarrow W, v \models_{\mathrm{D}} \psi\right)
\end{array}
$$

When an SD-model $W$ is fixed, the assignments $w \in W$ can be extended to truth assignments $w^{*}: \operatorname{FOR}\left(\mathcal{L}_{\mathrm{D}}\right) \rightarrow\{0,1\}$ in the natural way. The same, of course, applies to $\mathcal{L}_{\mathcal{U}}$.

Note that the truth definition of $\mathrm{D}\left(\varphi_{1}, \ldots, \varphi_{k} ; \varphi\right)$ extends the semantics of $\mathrm{D}$ in the logic $\mathcal{D}$ and does not here depend on the current state description $w$ but only on the entire SD-model $W$. In particular, the semantics of $C$ is as follows: $W, w \models \mathrm{C} \varphi$ iff for all $u, v \in W$, we have that $W, u \models \varphi$ iff $W, v \models \varphi$. In other words, $W, w \models \mathrm{C} \varphi$ iff the truth value of $\varphi$ is constant in the model.

Let us rephrase the semantic definition of $\mathrm{D}$ above in terms of explicit functional dependence. Every formula $\varphi \in F O R\left(\mathcal{L}_{\mathrm{D}}\right)$ determines the function $\mathrm{t}_{\varphi}^{W}: W \rightarrow\{0,1\}$ such that $\mathrm{t}_{\varphi}^{W}(w):=w^{*}(\varphi)$. Now we have $W, w \models \mathrm{D}\left(\varphi_{1}, \ldots, \varphi_{n} ; \varphi\right)$ if and only if $\mathrm{t}_{\varphi}^{W}$ is determined by $\mathrm{t}_{\varphi_{1}}^{W}, \ldots, \mathrm{t}_{\varphi_{n}}^{W}$ on $W$ in the sense of Definition 2.1, i.e.,

$$
W, w \models \mathrm{D}\left(\varphi_{1}, \ldots, \varphi_{n} ; \varphi\right) \text { iff } \operatorname{Det}_{W}\left(\mathrm{t}_{\varphi_{1}}^{W}, \ldots, \mathrm{t}_{\varphi_{n}}^{W} ; \mathrm{t}_{\varphi}^{W}\right) .
$$

The semantics of $\mathcal{L}_{\mathrm{D}}$ is similar to Kripke semantics (in the case where the accessibility relation of a model $W$ is the universal relation $W \times W$ ). Therefore it is natural to define for $\mathcal{L}_{\mathrm{D}}$ the notions of validity and satisfiability analogously to the corresponding definitions in modal logic:

Definition 3.1. Let $\varphi \in F O R\left(\mathcal{L}_{\mathrm{D}}\right)$.

1. $\varphi$ is valid in an $S D$-model $W$ if $W, w \models \varphi$ for every $w \in W$. We write $W \models \varphi$ if $\varphi$ is valid in $W$.

2. $\varphi$ is valid (or $S D$-valid), if $W \models \varphi$ for every SD-model $W$. We write $\models \varphi$ if $\varphi$ is valid.

3. $\varphi$ is satisfiable if $W, w \models \varphi$ for some SD-model $W \neq \emptyset$ and some $w \in W$.

The following definition is analogous to the definition of local equivalence in modal logic:

Definition 3.2. Let $\varphi$ and $\psi$ be formulae of $\mathcal{L}_{\mathrm{D}}$. We write $\varphi \equiv_{\mathcal{L}_{\mathrm{D}}} \psi$ if the equivalence $W, w \models \varphi \Leftrightarrow W, w \models \psi$ holds for all SD-models $W$ and all $w \in W$. We will often omit the subscript $\mathcal{L}_{\mathrm{D}}$ when no confusion arises.

\footnotetext{
${ }^{8}$ We sometimes write $W, w \models \mathcal{L}_{\mathrm{D}} \varphi$ instead or even simply $W, w \models \varphi$.
} 
We note that validity in an SD-model can be expressed locally in $\mathcal{L}_{\mathrm{D}}$ in the sense that for all assignments $w \in W$, we have $W \models \varphi$ iff $W, w \models \varphi \wedge C \varphi$. If we know that $W \neq \emptyset$, then $W \models \varphi$ iff $W, w \models \varphi \wedge C \varphi$ for some $w \in W$. Thus $\varphi \wedge C \varphi$ plays the role of the universal modality. Hence we define the notation $[\mathbf{u}] \varphi:=\varphi \wedge \mathrm{C} \varphi$ as well as $\langle\mathrm{u}\rangle \varphi:=\neg[\mathrm{u}] \neg \varphi$ (which can easily be seen to be equivalent to $\varphi \vee \neg \mathrm{C} \varphi$ ). Note that $[\mathrm{u}] \varphi$ and $\langle\mathrm{u}\rangle \varphi$ are simply abbreviations of formulae of $\mathcal{L}_{\mathrm{D}}$, they are not formulae of $\mathcal{L}_{\mathcal{U}}$. (The box and diamond operators in $\mathcal{L}_{\mathcal{U}}$ are denoted by $[\mathrm{U}]$ and $\langle\mathrm{U}\rangle$ instead of [u] and $\langle\mathbf{u}\rangle$.)

In turn, $\mathrm{D}$ is expressible in terms of $[\mathrm{u}]$ in the following sense:

\section{Proposition 3.3.}

$$
\mathrm{D}\left(\varphi_{1}, \ldots, \varphi_{k} ; \psi\right) \equiv \bigvee_{\chi \in D N F\left(\varphi_{1}, \ldots, \varphi_{k}\right)}[\mathrm{u}](\chi \leftrightarrow \psi)
$$

Proof. Let us denote the formula on the right hand side by $\Delta\left(\varphi_{1}, \ldots, \varphi_{k} ; \psi\right)$. Consider any SD-model $W$. Define $\llbracket \psi \rrbracket_{W}:=\{w \in W \mid W, w \models \psi\}$. For each $w \in W$, let $\chi_{w}$ be the unique type over $\left\{\varphi_{1}, \ldots, \varphi_{k}\right\}$ which is true at $w$; in the case $k=0$, let $\chi_{w}:=\top$. Define

$$
\chi_{(W, \psi)}:=\bigvee\left\{\chi_{w} \mid w \in \llbracket \psi \rrbracket_{W}\right\} .
$$

Now, suppose $W, w \models \mathrm{D}\left(\varphi_{1}, \ldots, \varphi_{k} ; \psi\right)$. We claim that $W, w \models[\mathrm{u}]\left(\chi_{(W, \psi)} \leftrightarrow \psi\right)$. Indeed, take any $v \in W$. If $W, v \models \psi$, then $v \in \llbracket \psi \rrbracket_{W}$ and $W, v \models \chi_{v}$ by definition, whence $W, v \models \chi_{(W, \psi)}$. Conversely, suppose $W, v \models \chi_{(W, \psi)}$. Then $W, v \models \chi_{u}$ for some $u \in \llbracket \psi \rrbracket_{W}$. Therefore $W, u \models \psi$ and $\chi_{v}=\chi_{u}$, i.e., for each $i=1, \ldots, k$, we have that $W, u \models \varphi_{i}$ iff $W, v \models \varphi_{i}$. Since $W, w \models \mathrm{D}\left(\varphi_{1}, \ldots, \varphi_{k} ; \psi\right)$, we thus have $W, u \models \psi$ iff $W, v \models \psi$. Therefore we infer that $W, v \models \psi$. Thus $W, v \models \chi_{(W, \psi)} \leftrightarrow \psi$ for every $v \in W$. Hence $W, w \models[\mathbf{u}]\left(\chi_{(W, \psi)} \leftrightarrow \psi\right)$, so $W, w \models \Delta\left(\varphi_{1}, \ldots, \varphi_{k} ; \psi\right)$.

Conversely, suppose $W, w \models \Delta\left(\varphi_{1}, \ldots, \varphi_{k} ; \psi\right)$. Thus $W, w \models[\mathrm{u}](\chi \leftrightarrow \psi)$ for some formula $\chi \in \operatorname{DNF}\left(\varphi_{1}, \ldots, \varphi_{k}\right)$. Suppose $u, v \in W$ are such that $W, u \models \varphi_{i}$ iff $W, v \models \varphi_{i}$ for each $i=1, \ldots, k$. Then $W, u \models \chi$ iff $W, v \models \chi$, whence $W, u \models \psi$ iff $W, v \models \psi$ because $W, u \models \chi \leftrightarrow \psi$ and $W, v \models \chi \leftrightarrow \psi$. Thus we have proved that $W, w \models \mathrm{D}\left(\varphi_{1}, \ldots, \varphi_{k} ; \psi\right)$.

Proposition 3.3 establishes that the operator $\mathrm{D}$ is definable in terms of $[\mathrm{u}]$. As a particular case of this definability, we obtain that $\mathbf{C} \psi \equiv[\mathbf{u}] \psi \vee[\mathbf{u}] \neg \psi$. Since D and $[\mathrm{u}]$ are interdefinable, it follows that the logics $\mathcal{L}_{\mathrm{D}}$ and $\mathcal{L}_{\mathcal{U}}$ are clearly equiexpressive: we can translate $\mathcal{L}_{\mathrm{D}}$ into $\mathcal{L}_{\mathcal{U}}$ by making use of Proposition 3.3 , and, on the other hand, we can translate $\mathcal{L}_{\mathcal{U}}$ into $\mathcal{L}_{\mathrm{D}}$ with the help of our earlier observation that the formula $\varphi \wedge \mathrm{C} \varphi$ simulates the universal modality. Below we will make significant use of this interdefinability of the operators $\mathrm{D}$ and $[\mathrm{u}]$.

The above results suggest obvious equivalence-preserving translations between $\mathcal{L}_{\mathrm{D}}$ and $\mathcal{L}_{\mathcal{U}}$. However, while this connection between $\mathcal{L}_{\mathrm{D}}$ and $\mathcal{L}_{\mathcal{U}}$ is interesting and useful, the primary aim of this paper is to study the notion of determinacy and the operator D taken as a primitive. Indeed, one of our principal objectives is to compare Propositional Dependence Logic $\mathcal{D}$ and the new logic $\mathcal{L}_{\mathrm{D}}$ and investigate how well 
they can be used in order to formalise statements about propositional determinacy and how well the two logics relate to natural language. The interdefinability of $\mathcal{L}_{\mathrm{D}}$ and $\mathcal{L}_{\mathcal{U}}$ can thus be regarded as an interesting fact that nevertheless will play mainly a technical role in this paper. Furthermore, neither of the above mentioned translations is polynomial, and in the more general framework of Kripke semantics (see Section 8.2 below) it is not always possible to define the box modality $\square$ in terms of the determinacy operator $\mathrm{D}$. We leave investigations of that more general framework for the future.

The following is a straightforward observation about $\mathcal{L}_{\mathrm{D}}$ which we will use later on.

Lemma 3.4. The logic $\mathcal{L}_{\mathrm{D}}$ satisfies the equivalent replacement property ER with respect to the equivalence given in Definition 3.2.

We say that a class $\mathcal{C}$ of SD-models is definable in $\mathcal{L}_{\mathrm{D}}$ (resp., in $\mathcal{L}_{\mathcal{U}}$ ), if there exists a formula $\varphi$ of $\mathcal{L}_{\mathrm{D}}$ (resp., of $\mathcal{L}_{\mathcal{U}}$ ) such that $W \models \varphi$ iff $W \in \mathcal{C}$.

Let $w: P R O P \rightarrow\{0,1\}$ be an assignment function, and let $\Phi \subseteq P R O P$. We let $\left.w\right|_{\Phi}$ denote the restriction of $w$ to $\Phi$, i.e., the function $f: \Phi \rightarrow\{0,1\}$ defined so that $f(p)=w(p)$ for all $p \in \Phi$. We define $\left.W\right|_{\Phi}:=\left\{\left.w\right|_{\Phi} \mid w \in W\right\}$.

Definition 3.5. Two SD-models $W_{1}$ and $W_{2}$ are $\Phi$-equivalent, denoted $W_{1} \equiv_{\Phi} W_{2}$, if $\left.W_{1}\right|_{\Phi}=\left.W_{2}\right|_{\Phi}$

Definition 3.6. A class of SD-models $\mathcal{C}$ is closed under finite propositional equivalence, if the following conditions hold.

1. $\emptyset \in \mathcal{C}$.

2. There exists a finite set $\Phi \subseteq P R O P$ such that for all nonempty SD-models $W_{1}$ and $W_{2}$, if $W_{1} \in \mathcal{C}$ and $W_{1} \equiv_{\Phi} W_{2}$, then $W_{2} \in \mathcal{C}$.

The first condition above has been included for technical convenience. Note that the empty model satisfies every formula of $\mathcal{L}_{\mathcal{U}}$ and $\mathcal{L}_{\mathrm{D}}$.

Proposition 3.7. A class of SD-models is definable in $\mathcal{L}_{\mathcal{U}}$ iff it is closed under finite propositional equivalence.

Proof. Suppose a class of SD-models $\mathcal{C}$ is definable in $\mathcal{L}_{\mathcal{U}}$ by some formula $\varphi$ and let $\Phi \subseteq P R O P$ be the set of proposition symbols that occur in $\varphi$. Then for all SD-models $W_{1}$ and $W_{2}$, if $W_{1} \in \mathcal{C}$ and $W_{1} \equiv_{\Phi} W_{2}$, then $W_{1} \models \varphi$ and thus $W_{2} \models \varphi$, whence $W_{2} \in \mathcal{C}$. Thus $\mathcal{C}$ is closed under finite propositional equivalence.

Now, suppose $\mathcal{C}$ is closed under finite propositional equivalence and let $\Phi \subseteq$ $P R O P$ be a finite set such that for all nonempty SD-models $W_{1}$ and $W_{2}$, if $W_{1} \in \mathcal{C}$ and $W_{1} \equiv_{\Phi} W_{2}$, then $W_{2} \in \mathcal{C}$. Define a characteristic formula $\varphi_{W}$ of a model $W \neq \emptyset$ as follows. For each $w \in W$, let $\chi_{w}$ be the unique propositional type in $\operatorname{Conj}(\Phi)$ such that $W, w \models \chi_{w}$. Define

$$
\varphi_{W}:=\left(\bigwedge_{w \in W}\langle\mathbf{u}\rangle \chi_{w}\right) \wedge[\mathbf{u}]\left(\bigvee_{w \in W} \chi_{w}\right),
$$


which of course can be regarded as a finite formula since we can eliminate repeated conjuncts and disjuncts.

Let

$$
\Delta(\Phi, \mathcal{C}):=\bigvee_{W \in \mathcal{C} \backslash\{\emptyset\}} \varphi_{W},
$$

which again can be regarded as a finite formula.

Then $\mathcal{C}$ is defined by $\Delta(\Phi, \mathcal{C})$. Indeed, $\Delta(\Phi, \mathcal{C})$ is true in every model $W \in \mathcal{C}$. Conversely, every SD-model $U \neq \emptyset$ satisfying $\Delta(\Phi, \mathcal{C})$ satisfies some disjunct, i.e., some characteristic formula $\varphi_{W}$ of some model $W \in \mathcal{C} \backslash\{\emptyset\}$, because the truth value of each disjunct of $\Delta(\Phi, \mathcal{C})$ is constant across the worlds of a given SD-model. Clearly $U \equiv_{\Phi} W$, whence we have $U \in \mathcal{C}$.

Consequently, we obtain a characterisation of the expressive power of $\mathcal{L}_{\mathrm{D}}$ :

Corollary 3.8. A class of SD-models is definable in $\mathcal{L}_{\mathrm{D}}$ iff it is closed under finite propositional equivalence.

Let $\Phi \neq \emptyset$ be a finite subset of PROP. Let $\mathcal{C}$ denote the set of all SD-models in restriction to $\Phi$, i.e., the set $\left\{\left.W\right|_{\Phi} \mid W\right.$ is an SD-model $\}$. We call $\mathcal{C}$ the set of $\Phi$-models and denote it by $\mathcal{M}(\Phi)$. Let $\mathcal{S} \subseteq \mathcal{M}(\Phi)$. We say that $\mathcal{S}$ is definable in $\mathcal{L}_{\mathcal{U}}\left(\mathcal{L}_{\mathrm{D}}\right)$ in restriction to $\mathcal{M}(\Phi)$, if there is a formula $\varphi$ of $\mathcal{L}_{\mathcal{U}}\left(\mathcal{L}_{\mathrm{D}}\right)$ Such that for all $W \in \mathcal{M}(\Phi)$, we have $W \models \varphi$ iff $W \in \mathcal{S}$.

Proposition 3.9. $\mathcal{L}_{\mathcal{U}}$ and $\mathcal{L}_{\mathrm{D}}$ are expressively complete in the sense that for any finite nonempty $\Phi \subseteq P R O P$ and any $\mathcal{S} \subseteq \mathcal{M}(\Phi)$ with $\emptyset \in \mathcal{S}$, the set $\mathcal{S}$ is definable in restriction to $\mathcal{M}(\Phi)$ in both $\mathcal{L}_{\mathcal{U}}$ and $\mathcal{L}_{\mathrm{D}}$.

Proof. The claim for $\mathcal{L}_{\mathcal{U}}$ is established by an argument that is almost identical to the proof of Proposition 3.7. The claim for $\mathcal{L}_{\mathrm{D}}$ then follows by the equiexpressivity of the logics $\mathcal{L}_{\mathrm{D}}$ and $\mathcal{L}_{\mathcal{U}}$.

\section{Propositional Logic of Independence $\mathcal{L}_{\mid}$}

We have defined the logic of $\mathcal{L}_{\mathrm{D}}$ as an extension of propositional logic PL with the operator D. Next we introduce Propositional Logic of Independence $\mathcal{L}_{\mathrm{I}}$ which extends PL in a similar way, but now with the operator I instead of D. The logic $\mathcal{L}_{\mathrm{I}}$ relates to Propositional Independence Logic $\mathcal{I}$ analogously to the way $\mathcal{L}_{\mathrm{D}}$ relates to Propositional Dependence Logic $\mathcal{D}$.

The language of Propositional Logic of Independence $\mathcal{L}_{\mathrm{I}}$ is given by the following grammar.

$$
\varphi::=p|\neg \varphi|(\varphi \rightarrow \varphi) \mid(\varphi, \ldots, \varphi) \mathrm{I}_{(\varphi, \ldots, \varphi)}(\varphi, \ldots, \varphi),
$$

where $p \in P R O P$, and each of the three tuples $(\varphi, \ldots, \varphi)$ in the expression

$$
(\varphi, \ldots, \varphi) \mathrm{I}_{(\varphi, \ldots, \varphi)}(\varphi, \ldots, \varphi)
$$

is a finite tuple of formulae; the tuples in the same expression may be of different lengths, but only the tuple in the subscript may possibly be empty. Instead of 
writing $(\varphi, \ldots, \varphi) \mathrm{I}_{\emptyset}(\varphi, \ldots, \varphi)$, we simply write $(\varphi, \ldots, \varphi) \mathrm{I}(\varphi, \ldots, \varphi)$. As in $\mathcal{L}_{\mathrm{D}}$ we consider the Boolean connectives $\wedge, \vee, \leftrightarrow$ definable as usual. Sometimes we leave out brackets of formulae of $\mathcal{L}_{\mathrm{l}}$, following the convention that the operator I has a higher priority than all binary connectives, while negation has a higher priority than all other operators (including I). We let $\operatorname{FOR}\left(\mathcal{L}_{\mathrm{I}}\right)$ denote the set of formulae of $\mathcal{L}_{1}$.

The semantics of $\mathcal{L}_{\mathrm{I}}$ is similar to that of $\mathcal{L}_{\mathrm{D}}$ and also defined with respect to SDmodels $W$ and assignments $w \in W$. Propositional symbols and Boolean operators are interpreted exactly as in $\mathcal{L}_{\mathrm{D}}$. To define the semantics for I, recall that in an SD-model $W$ and for a logic with a Kripke-style semantics, each $w \in W$ can be extended to a truth function $w^{*}$ from the set of formulae to $\{0,1\}$ such that $w^{*}(\varphi)=1$ iff $W, w \models \varphi$. The truth definition of the operator I extends the one in the logic $\mathcal{I}$ as follows. We define $W, w \models_{\mathcal{L}_{1}}\left(\varphi_{1}, \ldots, \varphi_{k}\right) \mathrm{I}_{\left(\theta_{1}, \ldots, \theta_{m}\right)}\left(\psi_{1}, \ldots, \psi_{n}\right)$ iff for all $w_{1}, w_{2} \in W$ that agree on $\theta_{1}, \ldots, \theta_{m}$ (i.e., are such that $w_{1}^{*}\left(\theta_{i}\right)=w_{2}^{*}\left(\theta_{i}\right)$ for all $i \in\{1, \ldots, m\})$, there exists some $v \in W$ such that

$$
\left(\bigwedge_{i \leq m} v^{*}\left(\theta_{i}\right)=w_{1}^{*}\left(\theta_{i}\right) \wedge \bigwedge_{i \leq k} v^{*}\left(\varphi_{i}\right)=w_{1}^{*}\left(\varphi_{i}\right) \wedge \bigwedge_{i \leq n} v^{*}\left(\psi_{i}\right)=w_{2}^{*}\left(\psi_{i}\right)\right) .
$$

Thus the operator I of $\mathcal{L}_{1}$ extends I of $\mathcal{I}$ so that in $\mathcal{L}_{1}$ the operator can be applied to all formulae, not only proposition symbols. Note that the semantics of I (which we defined with respect to the model $W$ and world $w \in W$ ) does not directly depend on the world $w$ but is global in the model.

Analogously to the conventions fixed in Definition 3.1 for $\mathcal{L}_{\mathrm{D}}$, we say that a formula $\varphi$ of $\mathcal{L}_{\mid}$is valid in a model $\mathrm{W}$ (denoted $W \models_{\mathcal{L}_{1}} \varphi$ ), if $\varphi$ is true in every world of $W$, and that $\varphi$ is valid (denoted $\models_{\mathcal{L}_{1}} \varphi$ ) if $W \models_{\mathcal{L}_{1}} \varphi$ for every model $W$. Two formulae $\varphi$ and $\psi$ of $\mathcal{L}_{1}$ are equivalent, denoted $\varphi \equiv_{\mathcal{L}_{1}} \psi$, if the equivalence $W, w \models_{\mathcal{L}_{1}} \varphi \Leftrightarrow W, w \models_{\mathcal{L}_{1}} \psi$ holds for all models $W$ and all $w \in W$.

The following two lemmas are straightforward to prove. For Lemma 4.2, recall the definition of $\Phi$-equivalence of SD-models from Definition 3.5 .

Lemma 4.1. The logic $\mathcal{L}_{\mathrm{I}}$ satisfies the equivalent replacements property ER with respect to $\equiv \mathcal{L}_{1}$.

Lemma 4.2. Let $\varphi$ be a formula of $\mathcal{L}_{\mathrm{D}}$ or $\mathcal{L}_{1}$. Let $\Phi$ be the set of proposition symbols occurring in $\varphi$. For nonempty models $W$ and $U$ and points $w \in W$ and $u \in U$, if $W \equiv_{\Phi} U$, then we have $W, w \models \varphi$ iff $U, u \models \varphi$.

Since both logics $\mathcal{L}_{\mathrm{D}}$ and $\mathcal{L}_{\mathrm{I}}$ are interpreted with respect to SD-models $W$ and assignments $w \in W$, it is easy to compare them. We define the following simple translation $t$ from $\mathcal{L}_{\mathrm{D}}$ into $\mathcal{L}_{\mathrm{l}}$ :

1. $t(p):=p$ for $p \in P R O P$

2. $t(\neg \varphi):=\neg t(\varphi)$

3. $t(\varphi \rightarrow \psi):=t(\varphi) \rightarrow t(\psi)$

4. $t\left(\mathrm{D}\left(\varphi_{1}, \ldots, \varphi_{k} ; \psi\right)\right):=t(\psi) \mathrm{I}_{\left(t\left(\varphi_{1}\right), \ldots, t\left(\varphi_{k}\right)\right)} t(\psi)$. 
In particular, we have $t(\mathrm{C} \psi):=t(\psi) \mid t(\psi)$. (Recall our discussion in Section 2.6 concerning formulae of the type $p \mid p$.)

Now we make some simple but interesting observations.

Proposition 4.3. $\mathcal{L}_{\mathrm{D}}$ embeds into $\mathcal{L}_{\mathrm{I}}$ and $\mathcal{D}$ embeds into $\mathcal{I}$, in the following sense.

1. For each formula $\varphi$ of $\mathcal{L}_{\mathrm{D}}$, there exists a formula $\psi$ of $\mathcal{L}_{\mathrm{I}}$ equivalent to $\varphi$, i.e., such that $W, w \models \varphi$ iff $W, w \models \psi$ for all $W$ and all $w \in W$.

2. For each formula $\varphi$ of $\mathcal{D}$, there exists a formula $\psi$ of $\mathcal{I}$ equivalent to $\varphi$, i.e., such that $W \Vdash \varphi$ iff $W \Vdash \psi$ for all $W$.

Proof. The proof of the first claim is straightforward, using the translation $t$ defined above. The second claim is also straightforward, based on the obvious variant $t^{\prime}$ of the translation $t$ that keeps proposition symbols and Boolean connectives the same and translates $\mathrm{D}\left(p_{1}, \ldots, p_{k} ; q\right)$ to $q \mathrm{I}_{\left(p_{1}, \ldots, p_{k}\right)} q$.

We note that the translation from $\mathcal{D}$ into $\mathcal{I}$ mentioned in the above proof is well known from the literature on team semantics.

We define $\left[\mathrm{u}^{\prime}\right] \varphi$ to be an abbreviation for the formula $\varphi \wedge \varphi \mathrm{l} \varphi$ of $\mathcal{L}_{\mathrm{l}}$, and we let $\left\langle\mathbf{u}^{\prime}\right\rangle \varphi$ denote $\neg\left[\mathbf{u}^{\prime}\right] \neg \varphi$. Note that $\left[\mathbf{u}^{\prime}\right]$ corresponds to the universal modality in an obvious way. To see how the independence operator I can be expressed in terms of $\left\langle\mathbf{u}^{\prime}\right\rangle$, consider a formula $\left(\varphi_{1}, \ldots, \varphi_{k}\right) \mathbf{I}_{\left(\theta_{1}, \ldots, \theta_{m}\right)}\left(\psi_{1}, \ldots, \psi_{n}\right)$ of $\mathcal{L}_{\mathbf{l}}$. Recall the notation $\operatorname{Conj}(\Phi)$ from Section 2.2, including also the special case for $\Phi=\emptyset$ which stipulates that $\operatorname{Conj}(\emptyset)=\{\top\}$. Define

$$
B:=\operatorname{Conj}\left(\left\{\varphi_{1}, \ldots, \varphi_{k}\right\}\right) \times \operatorname{Conj}\left(\left\{\theta_{1}, \ldots, \theta_{m}\right\}\right) \times \operatorname{Conj}\left(\left\{\psi_{1}, \ldots, \psi_{n}\right\}\right) .
$$

\section{Proposition 4.4.}

$$
\begin{aligned}
\left(\varphi_{1}, \ldots, \varphi_{k}\right) \mathrm{I}_{\left(\theta_{1}, \ldots, \theta_{m}\right)}\left(\psi_{1}, \ldots, \psi_{n}\right) & \\
& \equiv_{\mathcal{L}_{1}} \bigwedge_{(\varphi, \theta, \psi) \in B}\left(\left(\left\langle\mathbf{u}^{\prime}\right\rangle(\theta \wedge \varphi) \wedge\left\langle\mathbf{u}^{\prime}\right\rangle(\theta \wedge \psi)\right) \rightarrow\left\langle\mathbf{u}^{\prime}\right\rangle(\theta \wedge \varphi \wedge \psi)\right) .
\end{aligned}
$$

Proof. It is easy to see that the bottom formula describes the semantics of the operator $\mathbf{I}$ in terms of $\left\langle\mathbf{u}^{\prime}\right\rangle$ in a rather direct way.

We will complete the expressivity analysis of the $\operatorname{logics} \mathcal{L}_{\mathrm{D}}, \mathcal{L}_{\mathrm{I}}, \mathcal{D}$, and $\mathcal{I}$ in Section 6 .

\section{Natural language and logics of determinacy and in- dependence}

In this section we interpret the logics $\mathcal{D}$ and $\mathcal{L}_{\mathrm{D}}$ in relation to natural language and compare their respective properties. We will not discuss $\mathcal{I}$ explicitly here, but since $\mathcal{I}$ is technically quite similar to $\mathcal{D}$, many of the observations below concerning $\mathcal{D}$ 
apply to $\mathcal{I}$ as well. Here we take $W \Vdash \varphi$ (respectively, $W \models \psi$ ) to mean that a sensible agent who considers $W$ to be the set of all possible scenarios, considers $\varphi$ (resp., $\psi$ ) to hold. This kind of reading of SD-models as information states is in line with the the intuitions of modal logic and also inquisitive logic.

The principal argument of the section is that $\mathcal{L}_{\mathrm{D}}$ is - at least in some important respects - a better match than propositional dependence logic $\mathcal{D}$ with natural language intuitions concerning statements about logical determinacy. It is sufficient for our purposes to consider formulae with only the connectives $\neg$ and $\vee$ together with determinacy assertions of the type $\mathrm{D}(p ; q)$. We make the assumption that the desirable natural language counterparts of $\neg$ and $\vee$ should always be "it is not the case that" and "or," respectively. Formulae $\mathrm{D}(p ; q)$ should correspond to assertions stating that "whether $P$ holds, determines whether $Q$ holds." Here $P$ and $Q$ denote suitable natural language interpretations of $p$ and $q$. A different kind of analysis would arise if, for example, $\neg$ was to be read as "it is never the case that" or "it is impossible that."

Our argument will proceed as follows. We first argue that the semantics of formulae of the type $p \vee q$ is a good match with natural language intuitions in both logics $\mathcal{L}_{\mathrm{D}}$ and $\mathcal{D}$. We then turn to examples concerning formulae of the type $\mathrm{D}(p ; q)$, and again argue that the semantics of both logics is reasonable. (In this context we also briefly discuss more complex formulae of the type $\mathrm{D}(p, q ; r)$, but this is not crucial from the point of view of our discussion.) We then argue that, despite both $p \vee q$ and $\mathrm{D}(p ; q)$ having a reasonable semantics in $\mathcal{D}$, formulae of the type $\mathrm{D}(p ; q) \vee \mathrm{D}\left(p^{\prime} ; q^{\prime}\right)$, which combine $\vee$ and $\mathrm{D}$, are problematic. In fact, we show this even for the formula $\mathrm{D}(p ; q) \vee \mathrm{D}(p ; q)$, where both disjuncts are the same. We then continue by arguing that $\mathcal{L}_{\mathrm{D}}$, in turn, gives natural interpretations for these problematic examples. Finally, we briefly discuss formulae of the type $\neg \mathrm{D}(p ; q)$.

As we saw in the previous section (cf. Proposition 2.3), team semantics is simply classical semantics lifted to the level of sets: if $\varphi$ is a formula of propositional logic $\mathrm{PL}$, then, in the setting of team semantics, $W \Vdash \varphi$ simply means that each world in $W$ satisifies $\varphi$. Even the semantics of disjunction $\vee$, which may appear strange at first, makes perfect sense from that perspective. Let us consider an example where team semantics seems to give a correct interpretation (from the natural language perspective) to the disjunctive formula $p \vee q$.

Consider the following propositions.

- "The patient has an ear infection", encoded by $p$.

- "The patient has high blood pressure", encoded by $q$.

Assume a set $W=\left\{w_{1}, w_{2}, w_{3}\right\}$ of possible scenarios has been identified by a clinician after inspecting a patient with vertigo, where

$$
w_{1}(p)=1, w_{1}(q)=0 ; \quad w_{2}(p)=0, w_{1}(q)=1 ; \quad w_{3}(p)=1, w_{3}(q)=1 .
$$

The set $W=\left\{w_{1}, w_{2}, w_{3}\right\}$ is assumed here to be the set of exactly all scenarios which the clinician considers possible. The clinician has informed the patient about his situation, so the patient also considers $W$ to be the set of all possible scenarios. 
To summarize the discussion with the patient, and to repeat what the situation is, the clinician then states to the patient:

"So, you have an ear infection or high blood pressure."

The clinician seems to be asserting that " $W \Vdash p \vee q$," i.e., that the set $W$ of all possible scenarios splits into worlds that satisfy $p$ and worlds that satisfy $q$. Thus team semantics works correctly here. The interpretation " $W \Vdash p$ or $W \Vdash q$ " has a different meaning, which is false in this case.

In this example, the assertion $p \vee q$ was made about the set $W$ of possible states of affairs, i.e., sets of assignments. Since team semantics is based on sets of assignments (rather than individual assignments), it is a natural framework for interpreting determinacy atoms $\mathrm{D}\left(p_{1}, \ldots, p_{k} ; q\right)$. We next give natural language examples that should convince the reader that the semantics of the operator $D$ given by $\mathcal{D}$ is a good match with intuitions concerning statements about propositional determinacy.

Consider now a scenario with two containers of water in two laboratory ovens. Fix the following propositions.

- "The temperature is over $100^{\circ}$ Celsius", denoted by $p$.

- "The water is boiling", denoted by $q$.

Assume the setting is encoded by the set $W=\left\{w_{1}, w_{2}\right\}$, with one possible world for each container, such that $w_{1}=\{p, q\}$ and $w_{2}=\emptyset$. By $w_{1}=\{p, q\}$ we of course mean that $w_{1}(p)=1$ and $w_{1}(q)=1$, and analogously, $w_{2}=\emptyset$ means that $w_{2}(p)=0$ and $w_{2}(q)=0$.

Consider the following assertion:

"Whether the temperature is over $100^{\circ}$ Celsius determines whether the water is boiling."

It is natural to interpret the assertion to mean that $W \Vdash \mathrm{D}(p ; q)$. Thus the semantics of $\mathrm{D}$ seems to work fine here. For another example, extend the above setting with a third oven $w_{3}$ with a water container and a new proposition $r$ which asserts that the air pressure in the oven is over 1 bar. Let $W=\left\{w_{1}, w_{2}, w_{3}\right\}$, where

$$
\begin{aligned}
& \text { - } w_{1}=\{p, q\} \\
& \text { - } w_{2}=\emptyset \\
& \text { - } w_{3}=\{p, r\}
\end{aligned}
$$

This time the temperature being over $100^{\circ}$ Celsius does not determine whether the water is boiling, i.e., $W \forall \mathrm{D}(p ; q)$, because both $w_{1}$ and $w_{3}$ satisfy $p$, but the two worlds disagree on the truth value of $q$. However, we do have that $W \Vdash \mathrm{D}(p, r ; q)$. By adding yet another world $w_{4}=\{p, q, r\}$ encoding a fourth oven, we end up with a laboratory where $W \forall \mathbf{D}(p, r ; q)$. 
A scenario where $\mathrm{D}(p, r ; q)$ seems to hold universally, i.e., in every correctly designed set $W$ of possible worlds, can be obtained for example by considering a setting where each world is associated with a balance scale and two equally heavy weights. Let $p$ encode the assertion that exactly one weight has been placed on the left tray of the balance scale, and $r$ the corresponding assertion concerning the right tray; $q$ is the assertion that the scale is in balance. Now, indeed, $\mathrm{D}(p, r ; q)$ holds for any collection of physically possible worlds, with $q$ being true exactly when either $p$ and $r$ are both true or when they are both false.

We have seen that team semantics works fine on simple disjunctive formulae $p \vee q$ and and determinacy statements $\mathrm{D}(p ; q)$. We next combine disjunctions and determinacy statements and show that this leads to problematic interpretations from the point of view of natural language (cf. [Kuu14]).

Let $p$ denote the assertion that the sun is shining and $q$ the assertion that it is winter. Consider a setting where $W=\left\{w_{1}, w_{2}, w_{3}, w_{4}\right\}$, with all possible distributions of truth values for $p$ and $q$ realized. Now clearly $W \forall \mathrm{D}(p ; q)$, so whether the sun is shining does not determine whether it is winter. However, now $W \Vdash \mathrm{D}(p ; q) \vee \mathrm{D}(p ; q)$ holds in $\mathcal{D}$. This seems strange. Consider the following translation of the formula $\mathrm{D}(p ; q) \vee \mathrm{D}(p ; q)$ into natural language.

"Whether the sun is shining determines whether it is winter, or, whether the sun is shining determines whether it is winter."

The intuitively correct interpretation of the above statement seems to be the (indeed false) assertion that

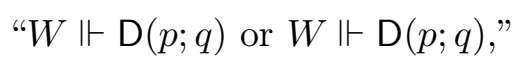

rather than the (true) assertion " $W \Vdash \mathrm{D}(p ; q) \vee \mathrm{D}(p ; q)$ " suggested by team semantics. The natural language statement "Whether the sun is shining determines whether it is winter, or, whether the sun is shining determines whether it is winter" seems obviously false. Therefore team semantics here gives an undesired interpretation to the formula $\mathrm{D}(p ; q) \vee \mathrm{D}(p ; q)$. In fact, we observe that the formula $\varphi:=\mathrm{D}(p ; q) \vee$ $\mathrm{D}(p ; q)$ is a validity of $\mathcal{D}$, i.e., we have $W \models \varphi$ for every SD-model $W$.

For another example, consider the formula $\mathrm{D}(p ; q) \vee \mathrm{D}(r ; q)$, where $p, q$ and $r$ stand for "It is dark", "John is at home", and "It is cold" respectively. Assume all distributions of truth values of the propositions $p, q, r$ are realized in $W$. Now $W \Vdash$ $\mathrm{D}(p ; q) \vee \mathrm{D}(r ; q)$ holds in $\mathcal{D}$. This is again counterintuitive from the natural language point of view. Like the formula $\mathrm{D}(p ; q) \vee \mathrm{D}(p ; q)$, also the formula $\mathrm{D}(p ; q) \vee \mathrm{D}(r ; q)$ is a validity of $\mathcal{D}$. In fact, every formula of the type $\mathrm{D}\left(p_{1}, \ldots, p_{k} ; q\right) \vee \mathrm{D}\left(r_{1}, \ldots, r_{n} ; q\right)$ is a validity of $\mathcal{D}$, because every SD-model $W$ can be split into sets $U, V \subseteq W$ such that $U \cup V=W$ and each assignment in $U$ satisfies $q$ while each assignment in $V$ satisfies $\neg q$.

Before we discuss how $\mathcal{L}_{\mathrm{D}}$ deals with the above formulae, we note once more that our analysis assumes that $\vee$ should correspond to the natural language "or." We do not want to claim that the natural language word or has always a unique 
interpretation 9 However, $\mathcal{L}_{\mathrm{D}}$ works quite nicely in the above examples, as we will next demonstrate.

Concerning the formula $p \vee q$ in the beginning of the section, we have $W \Vdash p \vee q$ iff $W \models p \vee q$. Also, for determinacy statements $\varphi:=\mathrm{D}\left(p_{1}, \ldots, p_{k} ; q\right)$, we have $W \Vdash \varphi$ iff $W \models \varphi$. Finally, for the problematic formulae $\mathrm{D}(p ; q) \vee \mathrm{D}(p ; q)$ and $\mathrm{D}(p ; q) \vee \mathrm{D}(r ; q)$, the semantics of $\mathcal{L}_{\mathrm{D}}$ gives the desired interpretations: we have $W \models \mathrm{D}(p ; q) \vee \mathrm{D}(p ; q)$ iff $(W \models \mathrm{D}(p ; q)$ or $W \models \mathrm{D}(p ; q))$ and similarly for the formula $\mathrm{D}(p ; q) \vee \mathrm{D}(r ; q)$.

We will not try to give an elaborated account of how well exactly $\mathcal{L}_{\mathrm{D}}$ corresponds to natural language, but it is essential to notice that $\mathcal{L}_{\mathrm{D}}$ can be considered to have a similar level of naturalness as standard S5 modal logic or modal logic with the universal modality. The reason for this is that a similar Kripke style semantics is used, and furthermore, it can be argued that $\mathcal{L}_{\mathrm{D}}$ is simply a fragment of the modal logic S5. This is because determinacy statements are naturally definable in terms of statements about possibility: simply consider the direct natural language translation of the equivalence

$$
\begin{aligned}
& \mathrm{D}(p ; q) \\
& \stackrel{\leftrightarrow}{\leftrightarrow}(\neg(\langle\mathbf{u}\rangle(p \wedge q) \wedge\langle\mathrm{u}\rangle(p \wedge \neg q)) \wedge \neg(\langle\mathbf{u}\rangle(\neg p \wedge q) \wedge\langle\mathbf{u}\rangle(\neg p \wedge \neg q))),
\end{aligned}
$$

where $\langle u\rangle$ should be read as "it is possible that." The natural language translation of this equivalence indeed seems intuitively immediately appealing, and importantly, the equivalence essentially just states the formal semantics of $\mathrm{D}(p ; q)$ (determinacy) in terms of the diamonds $\langle u\rangle$ (possibility), thus demonstrating that statements of determinacy can be very naturally and directly formulated in terms of possibility statements. Therefore $\mathcal{L}_{\mathrm{D}}$ can be considered a fragment of modal logic (with the universal or S5 modality in the particular case of this paper), and the level of correspondence between natural language and $\mathcal{L}_{\mathrm{D}}$ is similar to the corresponding relationship for (S5) modal logic. We note that the restriction to S5 frames is of no importance here. We also note that the above equivalence deals only with the simple determinacy formula $\mathrm{D}(p ; q)$ but it is easy to generalize our argument to more complex formulae. (See also Proposition 3.3.)

In addition to disjunction $\vee$, also the semantics of negation $\neg$ in $\mathcal{D}$ can be counterintuitive if the reading "it is not the case that" is desired for $\neg$. For example, let $p$ and $q$ denote the assertions "the Riemann hypothesis holds" and "it is raining," respectively. Let $W$ be a nonempty SD-model. Now we have $W \forall \neg \mathrm{D}(p ; q)$ in $\mathcal{D}$. In $\mathcal{L}_{\mathrm{D}}$, we have $W \models \neg \mathrm{D}(p ; q)$ in (for example) every model $W$ where the truth value of $p$ is the same in every possible world and where $q$ is true in some worlds and false in others 10

\footnotetext{
${ }^{9}$ There exist arguments essentially promoting the uniqueness of the meaning of disjunction (see Alo16 for an overview). On the other hand, already for example the inclusive and exclusive modes of or are sometimes taken to demonstrate ambiguity of disjunction. While we wish to refrain from taking any definite position in this debate, this issue is worth mentioning here. (We also want to point out that in the formal proofs and definitions of this article, the word or is used in the standard inclusive fashion as is customary in standard mathematical practice.)

${ }^{10}$ Perhaps alternative readings of $\neg$ could work better in $\mathcal{D}$. For example, in Kuu15, the reading "it is never the case that" for the negation of $\mathcal{D}$ is suggested. This reading is not meant
} 
Finally, concerning $\mathcal{L}_{\mathrm{D}}$, it is worth noting the triviality that just as in $\mathrm{S} 5, W \models$ $\neg \varphi$ is not in general equivalent to $W \not \models \varphi$, because $W \models \neg \varphi$ means that $W, w \not \models \varphi$ for all $w \in W$. Analogously, $W \models \varphi \vee \psi$ is not in general equivalent to ( $W \models$ $\varphi$ or $W \models \psi$ ). We note that, by Proposition 2.3 , we have $W \models \chi$ iff $W \Vdash \chi$ for all formulae of propositional logic in negation normal form, so $\mathcal{D}$ and $\mathcal{L}_{\mathrm{D}}$ are similar with respect to formulae of propositional logic PL.

To give an example of how the semantics of $\neg$ works in $\mathcal{D}$ and $\mathcal{L}_{\mathrm{D}}$ in the context of formulae of PL, let $p$ and $q$ denote the assertions "John has a cat" and "John is married," respectively. Assume a scenario where it is agreed that the possible worlds are $w_{1}=\{p\}$ and $w_{2}=\emptyset$, i.e., John may or may not have a cat, but he is definitely not married. Let $W=\left\{w_{1}, w_{2}\right\}$. The assertion "It is not the case that John is married" seems correct, and indeed, we have $W \Vdash \neg q$. Note, however, that even though $W \| p$, the claim "It is not the case that John has a cat" would seem odd. To make the claim $W \forall p$, one would have to assert, e.g., that it is possible that John does not have a cat, or that it is not necessarily the case that John has a cat.

\section{Comparing the expressive powers of $\mathcal{D}, \mathcal{I}, \mathcal{L}_{\mathrm{D}}$ and $\mathcal{L}_{\mathrm{l}}$}

We have earlier observed (Proposition 4.3) that $\mathcal{L}_{\mathrm{D}}$ embeds into $\mathcal{L}_{1}$, and also that $\mathcal{D}$ embeds into $\mathcal{I}$. In this section we will complete our discussion concerning the expressive powers of the logics $\mathcal{L}_{\mathrm{D}}, \mathcal{L}_{\mathrm{l}}, \mathcal{D}$ and $\mathcal{I}$.

\section{1 $\mathcal{I}$ does not embed into $\mathcal{D}$}

Let $\varphi, \psi \in F O R(\mathcal{D}) \cup F O R(\mathcal{I})$. We write $\varphi \equiv_{\text {team }} \psi$ if the equivalence $W \Vdash \varphi \Leftrightarrow$ $W \Vdash \psi$ holds for all SD-models $W$. The first result we wish to point out is wellknown; we prove it for the sake of completeness.

Proposition 6.1. The logic $\mathcal{I}$ does not embed into $\mathcal{D}$ : there exists a formula $\varphi$ of $\mathcal{I}$ such that for all formulae $\psi$ of $\mathcal{D}$, the equivalence $\varphi \equiv_{\text {team }} \psi$ fails.

Proof. It is well-known, and easy to show, that $\mathcal{D}$ satisfies the following downwards closure property: if $W \Vdash_{\mathcal{D}} \varphi$ and $U \subseteq W$, then we have $U \Vdash_{\mathcal{D}} \varphi$.

Now, define an SD-model $W=\left\{w_{1}, w_{2}, w_{3}, w_{4}\right\}$ where the four states represent all truth assignments for propositions $p$ and $q$. More precisely, let $w_{1}(p)=w_{1}(q)=$ $w_{2}(p)=w_{3}(q)=1$ and $w_{2}(q)=w_{3}(p)=w_{4}(p)=w_{4}(q)=0$. Define $U:=\left\{w_{1}, w_{4}\right\}$. Consider the formula $\varphi:=p \mid q$. It is clear that $W \Vdash \varphi$ and $U \| \varphi$. Therefore, due to the downwards closure property of $\mathcal{D}$, no formula $\psi$ of $\mathcal{D}$ can satisfy the equivalence $\varphi \equiv_{\text {team }} \psi$.

\subsection{Embedding $\mathcal{L}_{1}$ into $\mathcal{L}_{\mathrm{D}}$ via a concrete translation}

While $\mathcal{D}$ embeds into $\mathcal{I}$ but not vice versa, the situation is different for $\mathcal{L}_{\mathrm{D}}$ and $\mathcal{L}_{1}$. In Section 2.6 we established that $\mathcal{L}_{\mathrm{D}}$ embeds into $\mathcal{L}_{\mathrm{l}}$. We now define a translation

to necessarily have any temporal connotations, but instead could alternatively be read as "it is impossible that." We shall not try analyze here how well such a reading could actually work. 
showing that $\mathcal{L}_{\mathrm{I}}$ embeds into $\mathcal{L}_{\mathrm{D}}$, too. While it is straightforward to observe, based on Propositions 3.7 and 3.9, that $\mathcal{L}_{\mathrm{I}}$ indeed embeds into $\mathcal{L}_{\mathrm{D}}$, the concrete translation below will be of interest later on when we discuss compositional translations (to be defined) between the logics under investigation. We define the following translation $s: \operatorname{FOR}\left(\mathcal{L}_{\mathrm{l}}\right) \rightarrow \operatorname{FOR}\left(\mathcal{L}_{\mathrm{D}}\right):$

1. $s(p):=p$, for $p \in P R O P$

2. $s(\neg \varphi):=\neg s(\varphi)$

3. $s(\varphi \wedge \psi):=s(\varphi) \wedge s(\psi)$

4. We then translate the formula $\left(\varphi_{1}, \ldots, \varphi_{k}\right) \mathrm{I}_{\left(\theta_{1}, \ldots, \theta_{m}\right)}\left(\psi_{1}, \ldots, \psi_{n}\right)$ in a way that derives from Proposition 4.4. First we define

$$
\begin{aligned}
\mathcal{B}:=\operatorname{Conj}\left(\left\{s\left(\varphi_{1}\right), \ldots, s\left(\varphi_{k}\right)\right\}\right) \times \operatorname{Conj}\left(\left\{s\left(\theta_{1}\right)\right.\right. & \left.\left., \ldots, s\left(\theta_{m}\right)\right\}\right) \\
& \times \operatorname{Conj}\left(\left\{s\left(\psi_{1}\right), \ldots, s\left(\psi_{n}\right)\right\}\right) .
\end{aligned}
$$

For the special case where $m=0$, recall that $\operatorname{Conj}(\emptyset)=\{\top\}$. Now, let

$$
\begin{aligned}
& s\left(\left(\varphi_{1}, \ldots, \varphi_{k}\right) \mathrm{I}_{\left(\theta_{1}, \ldots, \theta_{m}\right)}\left(\psi_{1}, \ldots, \psi_{n}\right)\right):= \\
& \bigwedge_{(\varphi, \theta, \psi) \in \mathcal{B}}((\langle\mathrm{u}\rangle(\theta \wedge \varphi) \wedge\langle\mathrm{u}\rangle(\theta \wedge \psi)) \rightarrow\langle\mathrm{u}\rangle(\theta \wedge \varphi \wedge \psi)) .
\end{aligned}
$$

It is easy to see that the translation of the operator I given by the formula above describes the meaning of $\mathbf{I}$ quite directly in terms of $\langle\mathbf{u}\rangle$.

The proof of the next claim is straightforward, using the translation $s$ defined above.

Proposition 6.2. $\mathcal{L}_{\mathrm{I}}$ embeds into $\mathcal{L}_{\mathrm{D}}$ i.e., for each formula $\varphi$ of $\mathcal{L}_{\mathrm{l}}$, there exists an equivalent formula $\psi$ of $\mathcal{L}_{\mathrm{D}}$ in the sense that for all $W$ and all $w \in W$, we have $W, w \models \varphi$ iff $W, w \models \psi$.

\section{3 $\quad$ Strict embedding of $\mathcal{D}$ and $\mathcal{I}$ into $\mathcal{L}_{\mathrm{D}}$ and $\mathcal{L}_{\mathrm{I}}$}

Next we will show that the team-semantics-based logic $\mathcal{I}$ is strictly contained in the Kripke-style logic $\mathcal{L}_{\mathrm{D}}$ in the following sense.

1. For each formula $\varphi$ of $\mathcal{I}$, there exists a formula $\varphi^{\prime}$ of $\mathcal{L}_{\mathrm{D}}$ such that $\varphi$ and $\varphi^{\prime}$ define the same class of SD-models, i.e., $W \Vdash_{\mathcal{I}} \varphi$ iff $W \models \mathcal{L}_{\mathrm{D}} \varphi^{\prime}$ for all SD-models $W$.

2. There exists a formula $\psi$ of $\mathcal{L}_{\mathrm{D}}$ which is not equivalent to any formula of $\mathcal{I}$, i.e., for all formulae $\psi^{\prime}$ of $\mathcal{I}$, there exists a model $W$ such that $\left(W \Vdash \psi^{\prime}\right.$ and $W \not \models$ $\psi)$ or $\left(W \| \psi^{\prime}\right.$ and $\left.W \models \psi\right)$. 
The claim 1 above is essentially obvious, since all classes of models definable in $\mathcal{I}$ are closed under finite propositional equivalence, and due to Proposition [3.7, $\mathcal{L}_{\mathrm{D}}$ can define all such model classes. However, we will provide an explicit and effective translation of $\mathcal{I}$ into $\mathcal{L}_{\mathrm{D}}$ which is interesting in its own right and also elucidates the semantics of $\mathcal{I}$. Furthermore, despite the simplicity of our translation, we will show in Section 6.4 that there does not exist a compositional translation from $\mathcal{I}$ into $\mathcal{L}_{\mathrm{D}}$.

Recall once again the notion of a type normal form formula and related notions from Section 2, Let $\Phi$ be a finite nonempty set of proposition symbols and $\chi=$ $\bigvee\left\{\chi_{1}, \ldots, \chi_{k}\right\}$ a formula in $\operatorname{DNF}(\Phi)$. We let $\operatorname{SPLIT}(\chi)$ denote the set of pairs $(\alpha, \beta)$ of type normal form formulae in $\operatorname{DNF}(\Phi)$ such that if $\alpha=\bigvee\left\{\alpha_{1}, \ldots, \alpha_{m}\right\}$ and $\beta=\bigvee\left\{\beta_{1}, \ldots, \beta_{n}\right\}$, then we have

$$
\left\{\chi_{1}, \ldots, \chi_{k}\right\}=\left\{\alpha_{1}, \ldots, \alpha_{m}\right\} \cup\left\{\beta_{1}, \ldots, \beta_{n}\right\} .
$$

Let $\chi \in \operatorname{DNF}(\Phi)$. We define the following translation $t_{\chi}$ from $\mathcal{I}$ into $\mathcal{L}_{\mathrm{D}}$ :

1. $t_{\chi}(p):=[\mathbf{u}](\chi \rightarrow p)$ and $t_{\chi}(\neg p):=[\mathbf{u}](\chi \rightarrow \neg p)$

2. To translate the formula $\left(p_{1}, \ldots, p_{k}\right) \mathrm{I}_{\left(q_{1}, \ldots, q_{m}\right)}\left(r_{1}, \ldots, r_{n}\right)$, we use a suitably modified version of the equivalence in Proposition 4.4. We first define

$$
B:=\operatorname{Conj}\left(\left\{p_{1}, \ldots, p_{k}\right\}\right) \times \operatorname{Conj}\left(\left\{q_{1}, \ldots, q_{m}\right\}\right) \times \operatorname{Conj}\left(\left\{r_{1}, \ldots, r_{n}\right\}\right) .
$$

We then define $t_{\chi}\left(\left(p_{1}, \ldots, p_{k}\right) \mathrm{I}_{\left(q_{1}, \ldots, q_{m}\right)}\left(r_{1}, \ldots, r_{n}\right)\right):=$

$$
\bigwedge_{(\varphi, \psi, \theta) \in B}((\langle\mathbf{u}\rangle(\chi \wedge \psi \wedge \varphi) \wedge\langle\mathrm{u}\rangle(\chi \wedge \psi \wedge \theta)) \rightarrow\langle\mathrm{u}\rangle(\chi \wedge \psi \wedge \varphi \wedge \theta)) .
$$

3. $t_{\chi}(\varphi \wedge \psi):=t_{\chi}(\varphi) \wedge t_{\chi}(\psi)$

4. $t_{\chi}(\varphi \vee \psi):=\bigvee_{(\alpha, \beta) \in \operatorname{SPLIT}(\chi)}\left(t_{\alpha}(\varphi) \wedge t_{\beta}(\psi)\right)$

If $\varphi$ is a formula of $\mathcal{I}$ and $\Phi$ the set of proposition symbols in $\varphi$, we let $\chi(\varphi)$ denote the formula in $\operatorname{DNF}(\Phi)$ that contains as disjuncts all types over $\Phi$. Note that the formula $\chi(\varphi)$ is a tautology.

We then prove that our translation of $\mathcal{I}$ into $\mathcal{L}_{\mathrm{D}}$ preserves truth.

Lemma 6.3. $W \Vdash_{\mathcal{I} \varphi}$ iff $W \models \mathcal{L}_{\mathrm{D}} t_{\chi(\varphi)}(\varphi)$.

Proof. Let $\varphi$ be a formula of $\mathcal{I}$ and $W$ a model. Let $\Phi$ be the set of proposition symbols that occur in the formula $\varphi$. If $\chi \in D N F(\Phi)$, we let $W_{\chi}$ denote the set of worlds in $W$ that satisfy $\chi$. We will show that for every $\chi \in D N F(\Phi)$ and every subformula $\psi$ of $\varphi$, we have

$$
W_{\chi} \Vdash_{\mathcal{I}} \psi \text { iff } W \models \mathcal{L}_{\mathrm{D}} t_{\chi}(\psi) \text {. }
$$

The claim of the Lemma will then follow, as $W_{\chi(\varphi)}=W$. 
The proof proceeds by induction on the structure of $\psi$. The cases for proposition symbols, negated proposition symbols and conjunctions are straightforward. The argument for $\mathrm{I}$ is easy, as our translation in that case captures quite directly the semantics of $\mathrm{I}$.

We now proceed to the case $\psi=\psi^{\prime} \vee \psi^{\prime \prime}$. When going through the argument below, it helps to keep in mind the trivial technicality that for an SD-model $U$ and a proper subset $S$ of $P R O P$, there may exist several assignments in $U$ that are equivalent with respect to $S$, i.e., assignments that satisfy exactly the same propositions in $S$ (but differ elsewhere).

Assume that $W_{\chi} \Vdash_{\mathcal{I}} \psi^{\prime} \vee \psi^{\prime \prime}$. Thus there exist sets $S^{\prime}, S^{\prime \prime} \subseteq W_{\chi}$ such that $S^{\prime} \Vdash_{\mathcal{I}} \psi^{\prime}$ and $S^{\prime \prime} \Vdash_{\mathcal{I}} \psi^{\prime \prime}$, and furthermore, $S^{\prime} \cup S^{\prime \prime}=W_{\chi}$. Therefore there exists a pair $(\alpha, \beta) \in S P L I T(\chi)$ such that $S^{\prime} \subseteq W_{\alpha}$ and $S^{\prime \prime} \subseteq W_{\beta}$, and furthermore, $W_{\alpha} \equiv_{\Phi} S^{\prime}$ and $W_{\beta} \equiv_{\Phi} S^{\prime \prime}$ (recall Definition 3.5). Therefore clearly $W_{\alpha} \Vdash \psi^{\prime}$ and $W_{\beta} \Vdash \psi^{\prime \prime}$. Hence, by the induction hypothesis, we have $W \models_{\mathcal{L}_{\mathrm{D}}} t_{\alpha}\left(\psi^{\prime}\right)$ and $W \models \mathcal{L}_{\mathrm{D}} t_{\beta}\left(\psi^{\prime \prime}\right)$. Thus $W \models \mathcal{L}_{\mathrm{D}} t_{\alpha}\left(\psi^{\prime}\right) \wedge t_{\beta}\left(\psi^{\prime \prime}\right)$, whence we conclude that $W \models \mathcal{L}_{\mathrm{D}} t_{\chi}\left(\psi^{\prime} \vee \psi^{\prime \prime}\right)$.

For the converse, assume that $W \models \mathcal{L}_{\mathrm{D}} t_{\chi}\left(\psi^{\prime} \vee \psi^{\prime \prime}\right)$. Therefore there exist type normal form formulae $\alpha, \beta$ such that $(\alpha, \beta) \in S P L I T(\chi)$, and furthermore, $W \models \mathcal{L}_{\mathrm{D}}$ $t_{\alpha}\left(\psi^{\prime}\right)$ and $W \models \mathcal{L}_{\mathrm{D}} t_{\beta}\left(\psi^{\prime \prime}\right)$. By the induction hypothesis, we have $W_{\alpha} \Vdash_{\mathcal{I}} \psi^{\prime}$ and $W_{\beta} \Vdash_{\mathcal{I}} \psi^{\prime \prime}$. Since $(\alpha, \beta) \in \operatorname{SPLIT}(\chi)$, we have $W_{\alpha} \cup W_{\beta}=W_{\chi}$, and therefore $W_{\chi} \Vdash_{\mathcal{I}} \psi^{\prime} \vee \psi^{\prime \prime}$.

We are now ready to prove the following theorem.

Theorem 6.4. The logic $\mathcal{I}$ is strictly contained in $\mathcal{L}_{\mathrm{D}}$, i.e.:

1. For each $\varphi \in F O R(\mathcal{I})$, there exists a formula $\varphi^{\prime} \in F O R\left(\mathcal{L}_{\mathrm{D}}\right)$ (which can be found effectively), such that for all SD-models $W$, it holds that $W \Vdash \varphi$ iff $W \models \varphi^{\prime}$.

2. There exists a formula $\psi \in F O R\left(\mathcal{L}_{\mathrm{D}}\right)$ that is not expressible in $\mathcal{I}$, i.e., for all $\chi \in \mathcal{I}$, there exists an $S D$-model $W$ such that the equivalence $W \models \psi \Leftrightarrow W \Vdash \chi$ fails.

Proof. By Lemma 6.3 there exists an effective translation from $\mathcal{I}$ into $\mathcal{L}_{\mathrm{D}}$. Hence we only need to prove the second claim of the theorem. In fact the claim follows relatively easily from Proposition 3.7 and the proof of Theorem 4.2 of [KMSV15], but we will establish the claim here explicitly.

We will show that the $\mathcal{L}_{\mathrm{D}}$-formula $\neg \mathrm{C} p$ is not expressible in $\mathcal{I}$, i.e., there is no formula $\psi$ of $\mathcal{I}$ such that $W \vdash_{\mathcal{I}} \psi$ iff $W \models_{\mathcal{L}_{\mathrm{D}}} \neg \mathrm{C} p$. We first define two models $U$ and $U^{\prime}$, where $U$ consists of two worlds, one satisfying $p$ and the other one not, and $U^{\prime}$ consists of single world that does not satisfy $p$. Furthermore, for all other proposition symbols $q$, we define $q$ to be false in each world of the models $U, U^{\prime}$.

We then show by induction on the structure of formulae that for all $\varphi \in F O R(\mathcal{I})$, we have

$$
U \Vdash_{\mathcal{I}} \varphi \Rightarrow U^{\prime} \Vdash_{\mathcal{I}} \varphi
$$


For the literals $p$ and $\neg p$ this is immediate, as $U \forall_{\mathcal{I}} p$ and $U \forall_{\mathcal{I}} \neg p$. For other literals $q, \neg q$, etc., the implication holds because $U \forall_{\mathcal{I}} q$ and $U^{\prime} \Vdash_{\mathcal{I}} \neg q$. In order to deal with the operator $\mathbf{I}$, notice that $U^{\prime}$ satisfies all formulae of the type $\left(p_{1}, \ldots, p_{k}\right) \mathrm{I}_{\left(q_{1}, \ldots, q_{m}\right)}\left(r_{1}, \ldots, r_{n}\right)$, since the model $U^{\prime}$ contains only a single world.

The case for $\wedge$ follows immediately by the induction hypothesis.

We then consider the case for $\vee$. Assume that $U \vdash_{\mathcal{I}} \psi \vee \psi^{\prime}$. Therefore there exist sets $S, S^{\prime} \subseteq U$ such that $S \Vdash_{\mathcal{I}} \psi$ and $S^{\prime} \Vdash_{\mathcal{I}} \psi^{\prime}$, and furthermore, $S \cup S^{\prime}=U$. We may assume, by symmetry, that $S$ contains the assignment in $U$ that does not satisfy $p$. We consider two cases.

1. Assume that $S=U$. Then $U^{\prime} \Vdash_{\mathcal{I}} \psi$ follows directly by the induction hypothesis. Furthermore, we have $\emptyset \Vdash_{\mathcal{I}} \chi$ for every formula $\chi \in F O R(\mathcal{I})$, whence the condition

$$
U^{\prime} \Vdash_{\mathcal{I}} \psi \text { and } \emptyset \Vdash_{\mathcal{I}} \psi^{\prime}
$$

holds. Therefore $U^{\prime} \Vdash_{\mathcal{I}} \psi \vee \psi^{\prime}$.

2. Assume that $S$ is the singleton not satisfying $p$. Notice now that the world in $S$ and the world in $U^{\prime}$ satisfy exactly the same proposition symbols. Thus $S=U^{\prime}$, whence $U^{\prime} \Vdash_{\mathcal{I}} \psi$. Therefore the condition

$$
U^{\prime} \Vdash_{\mathcal{I}} \psi \text { and } \emptyset \Vdash_{\mathcal{I}} \psi^{\prime}
$$

holds again, and we hence conclude that $U^{\prime} \Vdash_{\mathcal{I}} \psi \vee \psi^{\prime}$.

Finally, note that $U \models \mathcal{L}_{\mathrm{D}} \neg \mathrm{C} p$, while $U^{\prime} \forall_{\mathcal{L}_{\mathrm{D}}} \neg \mathrm{C} p$. Therefore $\neg \mathrm{C} p$ cannot be expressible by a formula of $\mathcal{I}$.

Corollary 6.5. The logics $\mathcal{D}$ and $\mathcal{I}$ are both strictly contained in both $\mathcal{L}_{\mathrm{D}}$ and $\mathcal{L}_{1}$.

In summary, we have shown that $\mathcal{D}<\mathcal{I}<\mathcal{L}_{\mathrm{D}} \equiv \mathcal{L}_{\mathrm{l}}$, where $<$ denotes strict containment and $\equiv$ equi-expressivity. We have also observed that $\mathcal{L}_{\mathrm{D}}$ and $\mathcal{L}_{\mathrm{l}}$ are expressively complete in the sense that they can define exactly all classes of SDmodels closed under finite propositional equivalence. (See Definition [3.6] for the exact specification of finite propositional equivalence.)

\subsection{Regular logics and compositionality of translations}

Let $t^{*}$ denote the translation from $\mathcal{I}$ into $\mathcal{L}_{\mathrm{D}}$ we defined above. Recall that in addition to $t^{*}$, we have also defined the translations $t: F O R\left(\mathcal{L}_{\mathrm{D}}\right) \rightarrow F O R\left(\mathcal{L}_{\mathrm{I}}\right)$ and $s: \operatorname{FOR}\left(\mathcal{L}_{\mathrm{l}}\right) \rightarrow \operatorname{FOR}\left(\mathcal{L}_{\mathrm{D}}\right)$. Furthermore, in the proof of Proposition 4.3, we described a translation from $\mathcal{D}$ into $\mathcal{I}$; let us denote that translation by $t^{\prime}$.

In this section we will take a closer look at the four translations $t^{*}, t, s$ and $t^{\prime}$. We will establish that, in a certain sense, the translation $t^{*}$ is essentially different from the other three.

We begin by defining a notion of a syntactically regular logic suitable for our purposes. To this end, we first need some auxiliary definitions. Let $\mathbb{N}^{*}$ be the set of all finite sequences of numbers in $\mathbb{N}$ (including the empty sequence). Let $C$ be a finite or countably infinite set of operator symbols. Let $d$ be a function that associates with each symbol in $C$ a nonempty subset of $\mathbb{N}^{*}$; the set $d(c)$ is called 
the arity type set of $c$. For example, in $\mathcal{L}_{1}$, the operator I always operates on three tuples of formulae, with the middle tuple being the only one allowed to be the empty tuple, and thus the arity type set associated with I is $\mathbb{N}_{+} \times \mathbb{N} \times \mathbb{N}_{+}$.

The set $C$ together with the function $d$ give rise to a set $F O R(C, d)$ of formulae, which is defined to be the smallest set $S$ such that the following conditions hold:

1. If $p \in P R O P$, then $p \in S$.

2. If $c \in C,\left(n_{1}, \ldots, n_{k}\right) \in d(c)$, and $\varphi_{1,1}, \ldots, \varphi_{1, n_{1}}, \ldots, \varphi_{k, 1}, \ldots, \varphi_{k, n_{k}} \in S$, then

$$
c\left(\left(\varphi_{1,1}, \ldots, \varphi_{1, n_{1}}\right), \ldots,\left(\varphi_{k, 1}, \ldots, \varphi_{k, n_{k}}\right)\right) \in S .
$$

We call $F O R(C, d)$ the syntactically regular set of formulae defined by $C$ and $d$. We call a logic syntactically regular if the set of formulae of the logic is a syntactically regular set of formulae for some set $C$ and a related function $d$. Any logic whose set of formulae is a subformula closed subset of some syntactically regular set of formulae, is called a syntactically subregular logic. Closure of a formula set $F$ under subformulae obviously means that if $c\left(\left(\varphi_{1,1}, \ldots, \varphi_{1, n_{1}}\right), \ldots,\left(\varphi_{k, 1}, \ldots, \varphi_{k, n_{k}}\right)\right) \in F$, then each of the formulae $\varphi_{i, j}$ is in $F$. For the sake of simplicity, we will below mainly talk about regular and subregular (rather than syntactically regular and syntactically subregular) logics.

It is easy to see that $\mathcal{L}_{\mathrm{D}}$ and $\mathcal{L}_{\boldsymbol{1}}$ are essentially regular logics. Similarly, $\mathcal{D}$ and $\mathcal{I}$ are essentially subregular logics 11

Let $L$ be a subregular logic and $c$ an operator symbol of $L$. Let $d$ be the function that associates the operators of $L$ with the related arity type set, and let $x \in d(c)$. The pair $(c, x)$ is called a base operator of $L$. For example, the operator $\mathrm{D}$ of $\mathcal{L}_{\mathrm{D}}$ can act on tuples of formulae of all positive finite lengths, so each pair $(\mathrm{D}, i)$, where $i$ is a (singleton tuple containing a) positive integer, is a base operator. In contrast, the operators $\neg$ and $\rightarrow$ of $\mathcal{L}_{\mathrm{D}}$ are both associated with only a single arity type, and thus we can directly regard $\neg$ and $\rightarrow$ as base operators.

We now define the notion of a compositional translation from one subregular logic to another. Intuitively, a compositional translation from a logic $L$ to a logic $L^{\prime}$ has the property that each base operator of $L$ is described in $L^{\prime}$ in a uniform way. Therefore the translation in some sense acts only on the base operators of $L$ rather than directly on individual formulae. Thus a compositional translation can be considered to be, in a sense, simple and direct. For further discussion on compositional translations, see Jan97.

Assume $\varphi_{1}, \ldots, \varphi_{k}$, where $k \in \mathbb{N}$, are distinct formulae of a subregular logic $L$. Assume $\psi\left(\varphi_{1}, \ldots, \varphi_{k}\right)$ is a formula of $L$ obtained from $\varphi_{1}, \ldots, \varphi_{k}$ by composing these formulae with some collection of base operators. Let $X_{1}, \ldots, X_{k}$ be novel symbols. Then $\psi\left(X_{1}, \ldots, X_{k}\right)$ is called an operator term of $L$; the operator term is obtained by replacing the original ground instances of the formulae $\varphi_{1}, \ldots, \varphi_{k}$ in

\footnotetext{
${ }^{11}$ We acknowledge that strictly speaking $\mathcal{L}_{\mathrm{D}}$ and $\mathcal{L}_{\mathrm{I}}$ are not syntactically regular because- to give one reason - the connective $\rightarrow$ uses infix rather than prefix notation. However, it would be a trivial exercise to redefine the syntax of these logics in the required way, and therefore we consider them syntactically regular. Similarly, $\mathcal{D}$ and $\mathcal{I}$ are considered syntactically subregular.
} 
$\psi\left(\varphi_{1}, \ldots, \varphi_{k}\right)$ by $X_{1}, \ldots, X_{k}$, respectively. (For example, if $\varphi_{1}:=p$ and $\varphi_{2}:=q$, and if $\psi\left(\varphi_{1}, \varphi_{2}\right):=(p \rightarrow q) \vee p \mid q$, then we obtain the operator term $\left(X_{1} \rightarrow X_{2}\right) \vee X_{1} \mid X_{2}$ by replacing $\varphi_{1}$ with $X_{1}$ and $\varphi_{2}$ with $X_{2}$.)

Let $L$ and $L^{\prime}$ be subregular logics. We identify $L$ and $L^{\prime}$ with their respective sets of formulae. A translation $T: L \rightarrow L^{\prime}$ is compositional, if for each base operator $\left(c,\left(n_{1}, \ldots, n_{k}\right)\right)$ of $L$, there exists an operator term

$$
\psi\left(X_{1,1}, \ldots, X_{1, n_{1}}, \ldots, X_{k, 1}, \ldots, X_{k, n_{k}}\right)
$$

of $L^{\prime}$ such that for all tuples $\left(\varphi_{1,1}, \ldots, \varphi_{1, n_{1}}\right), \ldots,\left(\varphi_{k, 1}, \ldots, \varphi_{k, n_{k}}\right)$ of formulae of $L$ such that $c\left(\left(\varphi_{1,1}, \ldots, \varphi_{1, n_{1}}\right), \ldots,\left(\varphi_{k, 1}, \ldots, \varphi_{k, n_{k}}\right)\right) \in L$, we have

$$
\begin{aligned}
T\left(c\left(\left(\varphi_{1,1}, \ldots, \varphi_{1, n_{1}}\right), \ldots,\left(\varphi_{k, 1}, \ldots, \varphi_{k, n_{k}}\right)\right)\right) & \\
& :=\psi\left(T\left(\varphi_{1,1}\right), \ldots, T\left(\varphi_{1, n_{1}}\right), \ldots, T\left(\varphi_{k, 1}\right), \ldots, T\left(\varphi_{k, n_{k}}\right)\right),
\end{aligned}
$$

i.e., the translated formula is obtained by substituting each symbol $X_{i, j}$ in $\psi$ by $T\left(\varphi_{i, j}\right)$. Furthermore, it is required that for each proposition symbol $p$ in the syntax of $L$, the translation $T(p)$ contains no other proposition symbols except for $p$. This ensures that the translation $T(\varphi)$ of any formula $\varphi$ contains no other proposition symbols except for those in $\varphi$ itself. This is a natural requirement and can be essential for example when considering SD-models with a finite propositional signature, i.e, models that interpret only a finite number of proposition symbols. We note that in the team semantics literature, SD-models are in most cases indeed defined to interpret only finitely many proposition symbols.

The symbols $X_{i, j}$ in the above definition should be regarded as placeholders in the operator term $\psi\left(X_{1,1}, \ldots, X_{k, n_{k}}\right)$. Intuitively, the operator term $\psi\left(X_{1,1}, \ldots, X_{k, n_{k}}\right)$ provides a "uniform description" of the base operator $\left(c,\left(n_{1}, \ldots, n_{k}\right)\right)$ of $L$ in $L^{\prime}$. The above definition of a compositional translation is suitable for the purposes of the current paper and follows standard principles of compositional translations.

Note that our translations $t: F O R\left(\mathcal{L}_{\mathrm{D}}\right) \rightarrow F O R\left(\mathcal{L}_{\mathrm{I}}\right)$ and $s: F O R\left(\mathcal{L}_{\mathrm{I}}\right) \rightarrow$ $\operatorname{FOR}\left(\mathcal{L}_{\mathrm{D}}\right)$ are indeed compositional, as is the translation $t^{\prime}: \operatorname{FOR}(\mathcal{D}) \rightarrow \operatorname{FOR}(\mathcal{I})$ from the proof of Proposition 4.3. However, the translation $t^{*}: \operatorname{FOR}(\mathcal{I}) \rightarrow \operatorname{FOR}\left(\mathcal{L}_{\mathrm{D}}\right)$ is not compositional, despite being relatively simple. In fact, it will turn out that a sound compositional translation from $\mathcal{I}$ into $\mathcal{L}_{\mathrm{D}}$ or $\mathcal{L}_{\mathrm{l}}$ is not possible. To see this, it is sufficient (due to the existence of the translations $t^{\prime}$ and $s$ ) to show that $\mathcal{D}$ does not translate compositionally into $\mathcal{L}_{\mathrm{D}}$. The following theorem does exactly that.

Theorem 6.6. There exists no compositional translation $T$ from $\mathcal{D}$ into $\mathcal{L}_{\mathrm{D}}$ which is sound with respect to $S D$-models in the sense that for any $S D$-model $W$ and any formula $\varphi$ of $\mathcal{D}$, we have $W \Vdash \varphi$ iff $W \models T(\varphi)$.

Proof. Let $S$ be an SD-model and $\chi_{1}, \chi_{2}$ formulae of $\mathcal{L}_{\mathrm{D}}$. We say that $\chi_{1}$ and $\chi_{2}$ are locally equivalent in $S$, if for all $w \in S$, it holds that $S, w \models \chi_{1}$ iff $S, w \models \chi_{2}$.

Suppose, for the sake of contradiction, that a compositional translation $T$ from $\mathcal{D}$ into $\mathcal{L}_{\mathrm{D}}$ exists. Consider an SD-model $V$ consisting of exactly two assignments, one satisfying $p$ and the other one not. Then fix a proposition symbol $q$ so that for 
each $w \in V$, the assignment $w$ satisfies $q$ if and only if $w$ does not satisfy $p$. For all other proposition symbols $r \in P R O P \backslash\{p, q\}$, we assume that neither of the worlds in $V$ satisfies $r$. We then define an SD-model $U$ which is the same as $V$ but with the interpretation of $q$ redefined so that $q$ is satisfied by $u \in U$ if and only if $u$ satisfies $p$. Let $W \in\{U, V\}$. We will prove the following claims about $W$.

1. Let $r \in P R O P \backslash\{p, q\}$. The formula $T(r)$ is not satisfied by either of the points of $W$.

2. $T(q)$ is satisfied by exactly one point of $W$.

3. $T(\mathrm{C} p)$ is satisfied by exactly one point of $W$.

We begin with the first claim. If $T(r)$ was satisfied by both points of $W$, we would have $W \models T(r)$ and thus $W \Vdash r$, which is a contradiction. If $T(r)$ was satisfied by exactly one point of $W$, we would obtain a contradiction due to the fact that $T(r)$ is required by definition to contain no other proposition symbols except for $r$, and since $w(r)=0$ for both points $w \in W$, the situation where only one of the points in $W$ satisfies $T(r)$ is impossible by symmetry. Therefore the first claim holds.

Concerning the second claim, we first observe that $T(q)$ cannot be satisfied by both points of $W$, because if it was, then we would have $W \models T(q)$ and thus $W \Vdash q$, which is a contradiction. Now assume that neither of the points of $W$ satisfies $T(q)$. Then, using the first claim established above (claim 1.), the formulae $T(q)$ and $T(r)$, for $r \in P R O P \backslash\{p, q\}$, are locally equivalent in $W$. Therefore we can now infer, by the following argument, that the formulae $T(\mathrm{C} q)$ and $T\left(\mathrm{C}_{r}\right)$ must also be locally equivalent in $W$.

Since the translation $T$ is compositional, there exists an operator term $\psi(X)$ that describes the translation of the operator $\mathrm{C}$, and thus we have $T\left(\mathrm{C}_{q}\right)=\psi(T(q))$ and $T(\mathrm{Cr})=\psi(T(r))$. Since we know that $T(q)$ and $T(r)$ are locally equivalent in $W$, we immediately observe that $\psi(T(q))$ and $\psi(T(r))$ are also locally equivalent in $W$. Thus $T(\mathrm{C} q)$ and $T(\mathrm{C} r)$ are locally equivalent in $W$.

Hence, as $W \forall \mathrm{C} q$ and thus $W \forall T(\mathrm{C} q)$, we infer that $W \forall T(\mathrm{C} r)$. Therefore $W \| \mathrm{C} r$. This is a contradiction, and thus the second claim holds.

Concerning the third claim, assume first that $T(\mathrm{C} p)$ is satisfied by both points of $W$. Then $W \models T(\mathrm{C} p)$, whence $W \Vdash \mathrm{C} p$, which is a contradiction. Assume then that neither of the points in $W$ satisfies $T(\mathrm{C} p)$. Therefore, using the first claim (claim 1. above), $T(\mathrm{C} p)$ and $T(r)$ are locally equivalent in $W$. As $W \Vdash \mathrm{C} p \vee \mathrm{C}_{p}$, we have $W \models T(\mathrm{C} p \vee \mathrm{C} p)$ and thus $W \models \psi^{\prime}(T(\mathrm{C} p), T(\mathrm{C} p))$, where $\psi^{\prime}(X, Y)$ is the operator term for $\vee$ which demonstrates that $T$ is indeed a compositional translation. Since $T(\mathrm{C} p)$ and $T(r)$ are locally equivalent in $W$, we infer that $W \models \psi^{\prime}(T(r), T(r))$. Thus $W \Vdash r \vee r$, which is a contradiction. Therefore we conclude that $T(\mathrm{C} p)$ is satisfied by exactly one point of the model $W$, and hence the third claim holds.

We have now proved each of the above three claims. By the last two of the three claims, recalling that $T(\mathrm{C} p)$ can only use the proposition symbol $p$ and $T(q)$ the symbol $q$, we now observe that exactly one of the following conditions hold. 
1. $T(\mathrm{C} p)$ is locally equivalent to $T(q)$ in $U$.

2. $T(\mathrm{C} p)$ is locally equivalent to $T(q)$ in $V$.

We first assume that the first one of these conditions holds. Therefore, since $U \Vdash$ $\mathrm{C} p \vee \mathrm{C} p$ and thus $U \models T(\mathrm{C} p \vee \mathrm{C} p)$, we may now conclude that $U \models T(q \vee q)$ as follows.

We know that $T(C p)$ and $T(q)$ are satisfied by exactly the same single point in $U$. We know also that $U \models T(C p \vee C p)$. Thus $U \models \psi^{\prime}(T(C p), T(C p))$, where $\psi^{\prime}(X, Y)$ is the operator term for $\vee$. Since $T(C p)$ and $T(q)$ are locally equivalent in $U$, we therefore have $U \models \psi^{\prime}(T(q), T(q))$. Since $\psi^{\prime}(T(q), T(q))=T(q \vee q)$, we have $U \models T(q \vee q)$.

Since $U \models T(q \vee q)$, we have $U \Vdash q \vee q$, which is a contradiction. Thus we turn to the case where $T(\mathrm{C} p)$ and $T(q)$ are locally equivalent in $V$. Similarly to the above, since $V \Vdash \mathrm{C} p \vee \mathrm{C} p$ and thus $V \models T(\mathrm{C} p \vee \mathrm{C} p)$, we infer using the formula $\psi^{\prime}(X, Y)$ that $V \models T(q \vee q)$. Therefore $V \Vdash q \vee q$, which is a contradiction.

We finish this section by mentioning some relevant related results in the literature on compositional translations and uniform definability of operators. Section 3.5 of Cia09] establishes that in the propositional inquisitive logic InqL, which is a teambased logic equi-expressive with $\mathcal{D}$, none of the primitive operators is definable in terms of the others. In Yan16, it is shown that the implication and disjunction connectives of InqL are not uniformly definable in $\mathcal{D}$, and thus no compositional translation from InqL into $\mathcal{D}$ is possible. In Gal13, it is shown that the so-called weak universal quantifier $\forall^{1}$ is not uniformly definable in first-order dependence logic.

\section{$7 \quad$ Validities and axiomatizations}

In this section we provide sound and complete axiomatizations for $\mathcal{L}_{\mathrm{D}}$ and $\mathcal{L}_{\mathrm{I}}$. We begin by axiomatizing $\mathcal{L}_{\mathrm{C}}$, the fragment of $\mathcal{L}_{\mathrm{D}}$ with only operators $\mathrm{C}$ instead of general determinacy operators $\mathrm{D}$.

\subsection{Capturing the validities of $\mathcal{L}_{\mathrm{C}}$}

Recall that $\mathrm{C} \varphi$ stands for $\mathrm{D}(\epsilon ; \varphi)$, where $\epsilon$ is the empty sequence of formulae. Recall also the abbreviation $[\mathrm{u}] \varphi:=\varphi \wedge \mathrm{C} \varphi$ and the equivalence $\mathrm{C} \psi \equiv[\mathrm{u}] \psi \vee[\mathrm{u}] \neg \psi$ that intuitively demonstrate that the universal modality $[\mathrm{U}]$ and $\mathrm{C}$ are expressible in terms of each other.

We denote the fragment of $\mathcal{L}_{\mathrm{D}}$ that extends propositional logic PL with $\mathrm{C}$ by $\mathcal{L}_{\mathrm{C}}$. The operator $\mathrm{C}$ has been studied previously and in a more general setting as a "non-contingency" operator, and also - in epistemic logic - as a "knowing whether" operator, see [FWvD15] and the references therein.

We next present a sound and complete axiomatic system $A X\left(\mathcal{L}_{\mathrm{C}}\right)$ that captures the validities of $\mathcal{L}_{\mathrm{C}}$. Several proofs of completeness of equivalent axiomatizations have already been provided in the literature, starting with [MR66] and considered 
again in, e.g., Dem97. For historical references and recent related work on axiomatizations of C, see the above mentioned reference [FWvD15]. Nevertheless, we will present here yet another, simple and intuitive axiomatization with a purely syntactic proof of completeness by means of reduction to the completeness of S5 (or $\mathcal{L}_{\mathcal{U}}$ ). We will then use the completeness of $A X\left(\mathcal{L}_{\mathrm{C}}\right)$ to obtain complete axiomatizations for $\mathcal{L}_{\mathrm{D}}$ and $\mathcal{L}_{\mathrm{l}}$.

The axiomatic system $A X\left(\mathcal{L}_{\mathrm{C}}\right)$ is defined as follows.

Axiom schemes:

$\operatorname{Ax0}(C)$ A complete set of axioms for PL

$\operatorname{Ax} 1(C) C \top$

$\mathrm{Ax} 2(\mathrm{C}) \mathrm{C} \varphi \leftrightarrow \mathrm{C} \neg \varphi$

$\operatorname{Ax} 3(\mathrm{C}) \mathrm{C}(\varphi \wedge \mathrm{C} \varphi)$

$\mathrm{Ax} 4(\mathrm{C}) \mathrm{C} \varphi \wedge \mathrm{C} \psi \rightarrow \mathrm{C}(\varphi \wedge \psi)$

$\operatorname{Ax} 5(\mathrm{C}) \varphi \wedge \mathrm{C} \varphi \wedge \mathrm{C}(\varphi \rightarrow \psi) \rightarrow \mathrm{C} \psi$

Inference rules:

Rul0(C) Modus Ponens

$\operatorname{Rul1(C)}$ EQC: If $\vdash \varphi \leftrightarrow \psi$ then $\vdash \mathrm{C} \varphi \leftrightarrow \mathrm{C} \psi$.

We will denote derivability in $A X\left(\mathcal{L}_{\mathrm{C}}\right)$ by $\vdash_{\mathrm{C}}$.

The axiomatic system above is not minimal. For instance, $\operatorname{Ax} 4(\mathrm{C})$ can be left out 12 , as it is derivable (though, not quite trivially) from the others. Nevertheless, rather than providing a derivation of the axiom (which would not be in the focus of this paper), we prefer to keep it in the system.

Proposition 7.1. The following inference rule, which preserves SD-validity, can be used in $A X\left(\mathcal{L}_{\mathrm{C}}\right)$ :

$N E C_{\mathrm{C}}:$ If $\vdash_{\mathrm{C}} \varphi$ then $\vdash_{\mathrm{C}} \mathrm{C} \varphi$.

Proof. If $\vdash_{\mathrm{C}} \varphi$, then $\vdash_{\mathrm{C}} \varphi \leftrightarrow \top$ by PL (propositional logic). Thus $\vdash_{\mathrm{C}} \mathrm{C} \varphi \leftrightarrow \mathrm{C} \top$ by EQc, whence $\vdash_{\mathrm{C}} \mathrm{C} \varphi$ by using $\mathrm{Ax} 1(\mathrm{C})$ and PL.

Now, recall the following well-known complete S5 axiomatization for $\mathcal{L}_{\mathcal{U}}$.

Axiom schemata:

$\mathrm{Ax0}(\mathrm{U})$ A complete set of axioms for PL.

$\operatorname{Ax1}(\mathrm{U})[\mathrm{U}](\varphi \rightarrow \psi) \rightarrow([\mathrm{U}] \varphi \rightarrow[\mathrm{U}] \psi)$

$\operatorname{Ax} 2(\mathrm{U})[\mathrm{U}] \varphi \rightarrow \varphi$

\footnotetext{
${ }^{12}$ Thanks to Jie Fan for noting that.
} 
$\operatorname{Ax} 3(\mathrm{U})\langle\mathrm{U}\rangle \varphi \rightarrow[\mathrm{U}]\langle\mathrm{U}\rangle \varphi$

Inference rules:

Rul0(U) Modus Ponens

$\operatorname{Rul1}(\mathrm{U}) \mathrm{NEC}_{\mathrm{U}}:$ If $\vdash \varphi$ then $\vdash[\mathrm{U}] \varphi$

We will denote derivability in $\mathcal{L}_{\mathcal{U}}$ by $\vdash_{S 5}$.

To show completeness of $A X\left(\mathcal{L}_{\mathrm{C}}\right)$, we first extend the intuitive interdefinability of $\mathrm{C}$ and the universal modality to a translation $\varphi \mapsto \varphi^{+}$from $\mathcal{L}_{\mathrm{C}}$ into $\mathcal{L}_{\mathcal{U}}$ and a translation $\varphi \mapsto \varphi^{\circ}$ from $\mathcal{L}_{\mathcal{U}}$ into $\mathcal{L}_{\mathrm{C}}$.

The translation $\varphi \mapsto \varphi^{+}$from $\mathcal{L}_{\mathrm{C}}$ into $\mathcal{L}_{\mathcal{U}}$ is defined as follows.

1. $p^{+}=p$

2. $(\neg \varphi)^{+}=\neg \varphi^{+}$

3. $(\varphi \rightarrow \psi)^{+}=\left(\varphi^{+} \rightarrow \psi^{+}\right)$

4. $(\mathrm{C} \varphi)^{+}=[\mathrm{U}] \varphi^{+} \vee[\mathrm{U}] \neg \varphi^{+}$

The translation $\varphi \mapsto \varphi^{\circ}$ from $\mathcal{L}_{\mathcal{U}}$ into $\mathcal{L}_{\mathrm{C}}$ goes as follows.

1. $p^{\circ}=p$

2. $(\neg \varphi)^{\circ}=\neg \varphi^{\circ}$

3. $(\varphi \rightarrow \psi)^{\circ}=\left(\varphi^{\circ} \rightarrow \psi^{\circ}\right)$

4. $([\mathrm{U}] \varphi)^{\circ}=\left(\varphi^{\circ} \wedge \mathrm{C} \varphi^{\circ}\right)$

Lemma 7.2. For every formula $\varphi$ of $\mathcal{L}_{\mathrm{C}}, \models \varphi$ iff $\models \varphi^{+}$, where the validity statement in each case refers to the semantics of the language in question. Moreover, the translation ()$^{+}$preserves, both ways, truth in states and therefore validity in models.

The proof of the lemma is straightforward.

The composition of the two translations, first ()$^{+}$and then ()$^{\circ}$, defines the following translation $\varphi \mapsto \varphi^{*}$ from $\mathcal{L}_{\mathrm{C}}$ into $\mathcal{L}_{\mathrm{C}}$ :

1. $p^{*}=p$

2. $(\neg \varphi)^{*}=\neg \varphi^{*}$

3. $(\varphi \rightarrow \psi)^{*}=\left(\varphi^{*} \rightarrow \psi^{*}\right)$

4. $(\mathrm{C} \varphi)^{*}=\left(\varphi^{*} \wedge \mathrm{C} \varphi^{*}\right) \vee\left(\neg \varphi^{*} \wedge \mathrm{C} \neg \varphi^{*}\right)$

The following lemma shows that we can derive equivalence of $\varphi$ and its translation $\varphi^{*}$ in $A X\left(\mathcal{L}_{\mathrm{C}}\right)$.

Lemma 7.3. We have $\vdash_{\mathrm{C}} \varphi \leftrightarrow \varphi^{*}$ for every formula $\varphi \in \mathcal{L}_{\mathrm{C}}$. 
Proof. The proof proceeds by induction on the structure of $\varphi$. The case for atoms is trivial and the cases for the Boolean connectives follow easily from the induction hypothesis using the fact that $A X\left(\mathcal{L}_{\mathrm{C}}\right)$ is complete with respect to $\mathrm{PL}$. We thus discuss the case involving $\mathrm{C} \varphi$.

By the induction hypothesis, we have $\vdash_{\mathrm{C}} \varphi \leftrightarrow \varphi^{*}$. By the inference rule EQc, we obtain

$$
\vdash_{\mathrm{C}} \mathrm{C} \varphi \leftrightarrow \mathrm{C} \varphi^{*}
$$

On the other hand, by PL, we have

$$
\vdash_{\mathrm{C}} \mathrm{C} \varphi \leftrightarrow((\varphi \wedge \mathrm{C} \varphi) \vee(\neg \varphi \wedge \mathrm{C} \varphi)) .
$$

Then, again using PL together with the induction hypothesis and the equivalences above, we derive

$$
\vdash_{\mathrm{C}} \mathrm{C} \varphi \leftrightarrow\left(\left(\varphi^{*} \wedge \mathrm{C} \varphi^{*}\right) \vee\left(\neg \varphi^{*} \wedge \mathrm{C} \varphi^{*}\right)\right) .
$$

Finally, using the axiom $\mathrm{C} \theta \leftrightarrow \mathrm{C} \neg \theta$ with $\theta:=\varphi^{*}$ and PL, we get

$$
\vdash_{\mathrm{C}} \mathrm{C} \varphi \leftrightarrow\left(\left(\varphi^{*} \wedge \mathrm{C} \varphi^{*}\right) \vee\left(\neg \varphi^{*} \wedge \mathrm{C} \neg \varphi^{*}\right)\right)
$$

This concludes the proof.

Next we will show that every derivation in $\mathcal{L}_{\mathcal{U}}$ can be simulated by a derivation in $A X\left(\mathcal{L}_{\mathrm{C}}\right)$.

Lemma 7.4. For every formula $\varphi \in \mathcal{L}_{\mathcal{U}}$, if $\vdash_{\mathrm{S} 5} \varphi$ then $\vdash_{\mathrm{C}} \varphi^{\circ}$.

Proof. The proof proceeds by induction on derivations in $\mathcal{L}_{\mathcal{U}}$. We will first prove that $\vdash_{\mathrm{C}} \varphi^{\circ}$ for each axiom $\varphi$ for $\mathcal{L}_{\mathcal{U}}$. For propositional tautologies this is trivial.

To deal with $\operatorname{Ax} 1(\mathrm{U})$, we must show that

$$
\vdash_{\mathrm{C}}((\theta \rightarrow \psi) \wedge \mathrm{C}(\theta \rightarrow \psi)) \rightarrow((\theta \wedge \mathrm{C} \theta) \rightarrow(\psi \wedge \mathrm{C} \psi))
$$

for arbitrary $\theta$ and $\psi$. The following derivation does exactly this. (The steps after the first one use PL and the preceding steps.)

1. $\vdash_{\mathrm{C}}(\theta \wedge \mathrm{C} \theta \wedge \mathrm{C}(\theta \rightarrow \psi)) \rightarrow \mathrm{C} \psi$

2. $\vdash_{\mathrm{C}}(\theta \wedge \psi \wedge \mathrm{C} \theta \wedge \mathrm{C}(\theta \rightarrow \psi)) \rightarrow(\psi \wedge \mathrm{C} \psi)$

3. $\vdash_{\mathrm{C}}(\theta \wedge(\theta \rightarrow \psi) \wedge \mathrm{C} \theta \wedge \mathrm{C}(\theta \rightarrow \psi)) \rightarrow(\psi \wedge \mathrm{C} \psi)$

4. $\vdash_{\mathrm{C}}((\theta \rightarrow \psi) \wedge \mathrm{C}(\theta \rightarrow \psi) \wedge(\theta \wedge \mathrm{C} \theta)) \rightarrow(\psi \wedge \mathrm{C} \psi)$

5. $\vdash_{\mathrm{C}}((\theta \rightarrow \psi) \wedge \mathrm{C}(\theta \rightarrow \psi)) \rightarrow((\theta \wedge \mathrm{C} \theta) \rightarrow(\psi \wedge \mathrm{C} \psi))$

To cover axiom $\mathrm{Ax} 2(\mathrm{U})$, we must show that $\vdash_{\mathrm{C}}(\theta \wedge \mathrm{C} \theta) \rightarrow \theta$, which is a propositional tautology.

To deal with axiom $\mathrm{Ax} 3(\mathrm{U})$, we must show that

$$
\vdash_{\mathrm{C}} \neg(\neg \theta \wedge \mathrm{C} \neg \theta) \rightarrow(\neg(\neg \theta \wedge \mathrm{C} \neg \theta) \wedge \mathrm{C} \neg(\neg \theta \wedge \mathrm{C} \neg \theta)) .
$$

Here is the derivation. 
1. $\vdash_{\mathrm{C}} \neg(\neg \theta \wedge \mathrm{C} \neg \theta) \rightarrow \neg(\neg \theta \wedge \mathrm{C} \neg \theta)$

by $\mathrm{PL}$

2. $\vdash_{\mathrm{C}} \mathrm{C}(\neg \theta \wedge \mathrm{C} \neg \theta)$

$\operatorname{Ax} 3(\mathrm{C})$

3. $\vdash_{\mathrm{C}} \mathrm{C} \neg(\neg \theta \wedge \mathrm{C} \neg \theta)$

by row $2, \operatorname{Ax} 2(\mathrm{C})$ and $\mathrm{PL}$

4. $\vdash_{\mathrm{C}} \neg(\neg \theta \wedge \mathrm{C} \neg \theta) \rightarrow(\neg(\neg \theta \wedge \mathrm{C} \neg \theta) \wedge \mathrm{C} \neg(\neg \theta \wedge \mathrm{C} \neg \theta))$

by 1,3 and $\mathrm{PL}$

Now it remains to establish that $\mathrm{NEC}_{U}$ preserves the claim, that is, we will show that if $\vdash_{\mathrm{S} 5} \varphi$ and thus $\vdash_{\mathrm{C}} \varphi^{\circ}$ by the induction hypothesis, then we also have $\vdash_{\mathrm{C}}([\mathrm{U}] \varphi)^{\circ}$. Thus we assume that $\vdash_{\mathrm{C}} \varphi^{\circ}$. Using $\mathrm{NEC}_{\mathrm{C}}$ (see Proposition [7.1), we infer that $\vdash_{\mathrm{C}} \mathrm{C} \varphi^{\circ}$, and using $\mathrm{PL}$, we get $\vdash_{\mathrm{C}} \varphi^{\circ} \wedge \mathrm{C} \varphi^{\circ}$. As $([\mathrm{U}] \varphi)^{\circ}=\varphi^{\circ} \wedge \mathrm{C} \varphi^{\circ}$, we are done.

We are now ready to prove the soundness and completeness of $A X\left(\mathcal{L}_{\mathrm{C}}\right)$.

Proposition 7.5. The axiomatic system $A X\left(\mathcal{L}_{\mathrm{C}}\right)$ is sound and complete for the validities of $\mathcal{L}_{\mathrm{C}}$.

Proof. The soundness follows by an easy verification of the validity of all the axioms and the fact that EQc preserves validity.

To prove completeness, assume that $\models \varphi$ for some formula $\varphi$ of $\mathcal{L}_{\mathrm{C}}$. Then $\models \varphi^{+}$ by Lemma 7.2. By completeness of $\mathcal{L}_{\mathcal{U}}$, we have $\vdash_{S 5} \varphi^{+}$. By Lemma 7.4, we thus have $\vdash_{\mathrm{C}}\left(\varphi^{+}\right)^{\circ}$, i.e., $\vdash_{\mathrm{C}} \varphi^{*}$. Therefore $\vdash_{\mathrm{C}} \varphi$ by Lemma 7.3 .

\subsection{Complete axiomatizations of $\mathcal{L}_{\mathrm{D}}$ and $\mathcal{L}_{1}$}

We now define sound and complete axiomatic systems $A X\left(\mathcal{L}_{\mathrm{D}}\right)$ and $A X\left(\mathcal{L}_{\mathrm{l}}\right)$ for $\mathcal{L}_{\mathrm{D}}$ and $\mathcal{L}_{\mathrm{I}}$ by introducing new axiom schemata.

The axiomatic system $A X\left(\mathcal{L}_{\mathrm{D}}\right)$ for $\mathcal{L}_{\mathrm{D}}$ is obtained by extending $A X\left(\mathcal{L}_{\mathrm{C}}\right)$. The idea is simply to define $D$ in terms of $C$. A suitable definition is obtained from the equivalence established in Proposition 3.3 .

Recall that $[\mathbf{u}] \varphi$ is an abbreviation of $\varphi \wedge \mathrm{C} \varphi$. We define, for each positive integer $k$, the following axiom schema:

$$
A x\left(\mathrm{D}_{k}\right) \quad \mathrm{D}\left(\varphi_{1}, \ldots, \varphi_{k}, \psi\right) \leftrightarrow \bigvee_{\chi \in \operatorname{DNF}\left(\varphi_{1}, \ldots, \varphi_{k}\right)}[\mathrm{u}](\chi \leftrightarrow \psi)
$$

The system $A X\left(\mathcal{L}_{\mathrm{D}}\right)$ consists of the axiom schemata and rules of $\mathcal{L}_{\mathrm{C}}$ together with the above axiom schemata for each $k \in \mathbb{Z}_{+}$.

We obtain an axiomatic system $A X\left(\mathcal{L}_{\mathrm{l}}\right)$ for $\mathcal{L}_{\mathrm{l}}$ similarly by essentially extending $A X\left(\mathcal{L}_{\mathrm{C}}\right)$ by schemata that define by Proposition 4.4 the operator $\mathrm{I}$ in terms of $\mathrm{C}$. The language $\mathcal{L}_{1}$ does not contain $C$ as a primitive, but the translation $t$ given before Proposition 4.3 shows that the operator $C$ can be expressed as $\varphi \mathrm{I} \varphi$. Thus we first define $A X_{0}\left(\mathcal{L}_{\mathrm{l}}\right)$ to be the following system 13 obtained from $A X\left(\mathcal{L}_{\mathrm{C}}\right)$ by the substitution $\mathrm{C} \theta \mapsto \theta \mathbf{I} \theta$.

Axiom schemes:

\footnotetext{
${ }^{13}$ Provided here for readers' convenience.
} 
Ax0(I) A complete set of axioms for PL.

$\operatorname{Ax} 1(\mathrm{I}) \top \mathrm{I} \top$

$\operatorname{Ax} 2(\mathrm{I}) \varphi \mathrm{I} \varphi \leftrightarrow \neg \varphi \mathrm{I} \neg \varphi$

$\operatorname{Ax3}(\mathrm{I})(\varphi \wedge \varphi \mathrm{I} \varphi) \mathrm{I}(\varphi \wedge \varphi \mathrm{I} \varphi)$

$\operatorname{Ax} 4(\mathrm{I}) \varphi \mathrm{I} \varphi \wedge \psi \mathrm{I} \psi \rightarrow(\varphi \wedge \psi) \mathrm{I}(\varphi \wedge \psi)$

$\operatorname{Ax} 5(\mathrm{I}) \varphi \wedge \varphi \mathrm{I} \varphi \wedge(\varphi \rightarrow \psi) \mathrm{I}(\varphi \rightarrow \psi) \rightarrow \psi \mathrm{I} \psi$

Inference rules:

Rul0(C) Modus Ponens

$\operatorname{Rul1}(\mathrm{I}) \mathrm{EQ}_{\mathrm{I}}:$ If $\vdash \varphi \leftrightarrow \psi$ then $\vdash \varphi \mathrm{I} \varphi \leftrightarrow \psi \mathrm{I} \psi$.

Recalling the abbreviation $\left\langle u^{\prime}\right\rangle$ from Section 4, we define $A X\left(\mathcal{L}_{1}\right)$ to be the extension of $A X_{0}\left(\mathcal{L}_{\mathrm{l}}\right)$ by the following axiom schemata for all $m \in \mathbb{N}$ and $k, n \in \mathbb{Z}_{+}$:

$$
\begin{aligned}
& A x\left(\mathrm{I}_{k, m, n}\right) \quad\left(\varphi_{1}, \ldots, \varphi_{k}\right) \mathrm{I}_{\left(\theta_{1}, \ldots, \theta_{m}\right)}\left(\psi_{1}, \ldots, \psi_{n}\right) \leftrightarrow \\
& \bigwedge_{(\varphi, \theta, \psi) \in B}\left(\left(\left\langle\mathbf{u}^{\prime}\right\rangle(\theta \wedge \varphi) \wedge\left\langle\mathbf{u}^{\prime}\right\rangle(\theta \wedge \psi)\right) \rightarrow\left\langle\mathbf{u}^{\prime}\right\rangle(\theta \wedge \varphi \wedge \psi)\right),
\end{aligned}
$$

where $B$ is as in Proposition 4.4 .

We denote derivability in $A X\left(\mathcal{L}_{\mathrm{D}}\right)$ by $\vdash_{\mathrm{D}}$ and derivability in $A X\left(\mathcal{L}_{\mathrm{l}}\right)$ by $\vdash_{\mathrm{I}}$.

\section{Theorem 7.6.}

1. $A X\left(\mathcal{L}_{\mathrm{D}}\right)$ is sound and complete for the validities of $\mathcal{L}_{\mathrm{D}}$.

2. $A X\left(\mathcal{L}_{1}\right)$ is sound and complete for the validities of $\mathcal{L}_{1}$.

Proof. The proofs of the two claims are very similar, so we will first present the argument for $A X\left(\mathcal{L}_{\mathrm{D}}\right)$ and then briefly comment the claim for $A X\left(\mathcal{L}_{\mathrm{l}}\right)$.

Soundness follows from the soundness of $A X\left(\mathcal{L}_{\mathrm{C}}\right)$ and Proposition 3.3. To prove completeness, we will use a similar argument as the one applied in the proof of Proposition 7.5. We will reduce the completeness of $A X\left(\mathcal{L}_{\mathrm{D}}\right)$ to the already proved completeness of $A X\left(\mathcal{L}_{\mathrm{C}}\right)$.

We first define the obvious translation $t r$ of $\mathcal{L}_{\mathrm{D}}$ into $\mathcal{L}_{\mathrm{C}}$ which leaves all atoms and Boolean connectives intact and likewise translates $\mathrm{C} \varphi$ to $\mathrm{C} \operatorname{tr}(\varphi)$, but treats formulae $\mathrm{D}\left(\varphi_{1}, \ldots, \varphi_{k} ; \psi\right)$ with $k \neq 0$ as follows. Using the equivalence established by Proposition 3.3, we put

$$
\operatorname{tr}\left(\mathrm{D}\left(\varphi_{1}, \ldots, \varphi_{k} ; \psi\right)\right):=\bigvee_{\chi \in D N F\left(\varphi_{1}, \ldots, \varphi_{k}\right)}((\operatorname{tr}(\chi) \leftrightarrow \operatorname{tr}(\psi)) \wedge \mathrm{C}(\operatorname{tr}(\chi) \leftrightarrow \operatorname{tr}(\psi)))
$$


We then prove by induction on the structure of formulae $\theta$ of $\mathcal{L}_{\mathrm{D}}$ that

$$
\vdash_{\mathrm{D}} \theta \leftrightarrow \operatorname{tr}(\theta) .
$$

The cases for proposition symbols and Boolean connectives are trivial. To cover the case for $\mathrm{C}$, assume we have shown that $\vdash_{\mathrm{D}} \varphi \leftrightarrow t r(\varphi)$. We then conclude that $\vdash_{\mathrm{D}} \mathrm{C} \varphi \leftrightarrow \mathrm{C} \operatorname{tr}(\varphi)$ directly by the rule $\mathrm{EQ}_{\mathrm{C}}$.

To deal with the case for $\mathrm{D}$, let $\theta=\mathrm{D}\left(\varphi_{1}, \ldots, \varphi_{k} ; \psi\right)$, and let the induction hypothesis be that $\vdash_{\mathrm{D}} \varphi_{i} \leftrightarrow \operatorname{tr}\left(\varphi_{i}\right)$ for each $i \leq k$ and $\vdash_{\mathrm{D}} \psi \leftrightarrow \operatorname{tr}(\psi)$. From here it is easy to conclude, using completeness with respect to propositional logic, that we also have $\vdash_{\mathrm{D}} \chi \leftrightarrow \operatorname{tr}(\chi)$ for each $\chi \in \operatorname{DNF}\left(\varphi_{1}, \ldots, \varphi_{k}\right)$. Therefore, using PL, we have

$$
\vdash_{\mathrm{D}}(\chi \leftrightarrow \psi) \leftrightarrow(\operatorname{tr}(\chi) \leftrightarrow \operatorname{tr}(\psi))
$$

whence we infer by the rule $\mathrm{EQ}_{\mathrm{C}}$ that we have

$$
\vdash_{\mathrm{D}} \mathrm{C}(\chi \leftrightarrow \psi) \leftrightarrow \mathrm{C}(\operatorname{tr}(\chi) \leftrightarrow \operatorname{tr}(\psi)) .
$$

Using this equivalence and the already established fact that $\vdash_{\mathrm{D}} \chi^{\prime} \leftrightarrow \operatorname{tr}\left(\chi^{\prime}\right)$ for all $\chi^{\prime} \in\{\psi\} \cup \operatorname{DNF}\left(\varphi_{1}, \ldots, \varphi_{k}\right)$, we then infer by PL that

$$
\begin{aligned}
& \vdash_{\mathrm{D}} \bigvee_{\chi \in D N F\left(\varphi_{1}, \ldots, \varphi_{k}\right)}((\chi \leftrightarrow \psi) \wedge \mathrm{C}(\chi \leftrightarrow \psi)) \\
& \leftrightarrow \bigvee_{\chi \in D N F\left(\varphi_{1}, \ldots, \varphi_{k}\right)}((\operatorname{tr}(\chi) \leftrightarrow \operatorname{tr}(\psi)) \wedge \mathrm{C}(\operatorname{tr}(\chi) \leftrightarrow \operatorname{tr}(\psi))) .
\end{aligned}
$$

From here we conclude, using propositional logic and $\operatorname{Ax}\left(D_{k}\right)$, that

$$
\vdash_{\mathrm{D}} \mathrm{D}\left(\varphi_{1}, \ldots, \varphi_{k} ; \psi\right) \text { } \leftrightarrow \bigvee_{\chi \in D N F\left(\varphi_{1}, \ldots, \varphi_{k}\right)}((\operatorname{tr}(\chi) \leftrightarrow \operatorname{tr}(\psi)) \wedge \mathrm{C}(\operatorname{tr}(\chi) \leftrightarrow \operatorname{tr}(\psi))) .
$$

In other words, we have $\vdash_{\mathrm{D}} \mathrm{D}\left(\varphi_{1}, \ldots, \varphi_{k}, \psi\right) \leftrightarrow \operatorname{tr}\left(\mathrm{D}\left(\varphi_{1}, \ldots, \varphi_{k}, \psi\right)\right)$, whence we have now established that $\vdash_{\mathrm{D}} \theta \leftrightarrow \operatorname{tr}(\theta)$ for all $\theta$ of $\mathcal{L}_{\mathrm{D}}$.

To conclude the proof, assume that $\models \theta$ for some $\theta$ of $\mathcal{L}_{\mathrm{D}}$. Then $\models \operatorname{tr}(\theta)$ by soundness of the translation $\operatorname{tr}$. Hence, recalling that $\operatorname{tr}(\theta)$ is a formula of $\mathcal{L}_{\mathrm{C}}$, we have $\vdash_{\mathrm{C}} \operatorname{tr}(\theta)$ by completeness of $A X\left(\mathcal{L}_{\mathrm{C}}\right)$. Using the fact that $\vdash_{\mathrm{D}} \theta \leftrightarrow \operatorname{tr}(\theta)$, we extend the derivation of $\operatorname{tr}(\theta)$ in $A X\left(\mathcal{L}_{\mathrm{C}}\right)$ to a derivation of $\theta$ in $A X\left(\mathcal{L}_{\mathrm{D}}\right)$. Therefore $\vdash_{\mathrm{D}} \theta$.

The completeness proof of $A X\left(\mathcal{L}_{\mathrm{l}}\right)$ is similar. We first prove that $A X_{0}\left(\mathcal{L}_{\mathrm{l}}\right)$ is complete for the notational variant of $\mathcal{L}_{\mathrm{C}}$ that replaces $\mathrm{C} \varphi$ with $f(\varphi) \mid f(\varphi)$, where $f$ is a translation that keeps proposition variables and Boolean connectives intact but treats $\mathrm{C}$ as given here. This proof of completeness is virtually identical to the corresponding argument for $A X\left(\mathcal{L}_{\mathrm{C}}\right)$ given above. Then the completeness of $A X\left(\mathcal{L}_{\mathrm{l}}\right)$ is proved similarly to the way $A X\left(\mathcal{L}_{\mathrm{D}}\right)$ was treated above, the only significant (but uncomplicated) difference being that the axioms $A x\left(\mathrm{I}_{k, m, n}\right)$ instead of axioms $A x\left(\mathrm{D}_{k}\right)$ are used. 
Our axiomatizations for $\mathcal{L}_{\mathrm{D}}$ and $\mathcal{L}_{\mathrm{I}}$ are not finite because we have the schemata $A x\left(\mathrm{D}_{k}\right)$ and $A x\left(\mathrm{I}_{k, m, n}\right)$ for infinitely many values of $k, m, n$.14 We will next show that, in fact, neither $\mathcal{L}_{\mathrm{D}}$ nor $\mathcal{L}_{\mathrm{I}}$ has a finite axiomatization. To this end, we will first define formally what we mean by a finite axiomatization.

An axiom schema for $\mathcal{L}_{\mathrm{D}}$ (respectively, $\mathcal{L}_{\mathrm{I}}$ ) is an object obtained from a formula $\chi$ of $\mathcal{L}_{\mathrm{D}}\left(\mathcal{L}_{\mathrm{I}}\right)$ by substituting schema letters $\varphi_{1}, \ldots, \varphi_{n}$ for all proposition symbols in $\chi$. A proof rule for $\mathcal{L}_{\mathrm{D}}\left(\mathcal{L}_{1}\right)$ is an implication of the form $\vdash \psi_{1}, \ldots, \vdash \psi_{k} \Rightarrow \vdash \chi$, where $\psi_{1}, \ldots, \psi_{k}, \chi$ are axiom schemata for $\mathcal{L}_{\mathrm{D}}\left(\mathcal{L}_{1}\right)$ and $k$ a positive integer. A finite axiomatization for $\mathcal{L}_{\mathrm{D}}\left(\mathcal{L}_{\mathrm{l}}\right)$ is a pair $(\Phi, \Psi)$, where $\Phi$ is a finite set of axiom schemata and $\Psi$ a finite set of proof rules for $\mathcal{L}_{\mathrm{D}}\left(\mathcal{L}_{1}\right)$.

Theorem 7.7. Neither $\mathcal{L}_{\mathrm{D}}$ nor $\mathcal{L}_{\mathrm{I}}$ has a sound and complete finite axiomatization.

Proof. We discuss $\mathcal{L}_{\mathrm{D}}$ only. The argument for $\mathcal{L}_{\mathrm{I}}$ is similar.

Assume $(\Phi, \Psi)$ is a sound and complete finite axiomatization for $\mathcal{L}_{\mathrm{D}}$. Let $k$ be the maximum number such that some schema in $\Phi \cup \Psi$ contains a subschema of the type $\mathrm{D}\left(\psi_{1}, \ldots, \psi_{k} ; \varphi\right)$. For each formula $\chi$ of $\mathcal{L}_{\mathrm{D}}$, let $\chi(\perp)$ denote the formula obtained from $\chi$ by replacing (in any order) each subformula of the type $\mathrm{D}\left(\alpha_{1}, \ldots, \alpha_{k+1} ; \beta\right)$ by $\perp$. We will show by induction on deductions that for all formulae $\chi$ of $\mathcal{L}_{\mathrm{D}}$, if $\chi$ is a theorem of $(\Phi, \Psi)$, then also $\chi(\perp)$ is a theorem of $(\Phi, \Psi)$. This will conclude the proof for the following reason. Consider the formula $\mathrm{D}(p, \ldots, p ; p)$, where $p$ is simply repeated $k+2$ times. This formula is a theorem of $\mathcal{L}_{\mathrm{D}}$, while $\perp$ is not.

The inductive argument is based on the following observation: if $\alpha$ is a formula obtained from a schema $\varphi$ by substitution, then also the formula $\alpha(\perp)$ can be obtained from $\varphi$ by substitution, because the schema $\varphi$ does not involve any subschemata of the type $\mathrm{D}\left(\chi_{1}, \ldots, \chi_{k+1} ; \chi^{\prime}\right)$. Therefore the basis of the induction, which deals with the direct use of axiom schemata as a first step of a deduction, is clear. The induction step is based on similar reasoning. For consider a proof rule $\vdash \varphi_{1}, \ldots, \vdash \varphi_{m} \Rightarrow \vdash \psi$ and assume that we have deduced some formula $\beta$ by applying an instance $\vdash \alpha_{1}, \ldots, \vdash \alpha_{m} \Rightarrow \vdash \beta$ of this rule to some formulae $\alpha_{1}, \ldots, \alpha_{m}$ such that $\vdash \alpha_{1}, \ldots, \vdash \alpha_{m}$. Since the schemata $\varphi_{1}, \ldots, \varphi_{m}, \psi$ do not contain subschemata of the type $\mathrm{D}\left(\chi_{1}, \ldots, \chi_{k+1}, \chi^{\prime}\right)$, we observe that the implication $\vdash \alpha_{1}(\perp), \ldots, \vdash \alpha_{m}(\perp) \Rightarrow$ $\vdash \beta(\perp)$ is also an instance of the rule $\vdash \varphi_{1}, \ldots, \vdash \varphi_{m} \Rightarrow \vdash \psi$. Since $\vdash \alpha_{1}, \ldots, \vdash \alpha_{m}$, we have $\vdash \alpha_{1}(\perp), \ldots, \vdash \alpha_{m}(\perp)$ by the induction hypothesis. Thus $\vdash \beta(\perp)$, as required.

\section{The road ahead}

In this paper we have defined the logics $\mathcal{L}_{\mathrm{D}}$ and $\mathcal{L}_{\mathrm{I}}$ as alternatives for $\mathcal{D}$ and $\mathcal{I}$. We have comprehensively studied the expressive powers of these four logics and argued for the naturalness of $\mathcal{L}_{\mathrm{D}}$ and $\mathcal{L}_{\mathrm{l}}$ in relation to $\mathcal{D}$ and $\mathcal{I}$. We have also provided sound and complete axiomatizations for $\mathcal{L}_{\mathrm{D}}$ and $\mathcal{L}_{\mathrm{l}}$. Here we discuss briefly a range of natural future developments of the present work.

\footnotetext{
${ }^{14}$ Indeed, bounding these values by some small constant would result in more elegant axiomatizations that then would, however, only work for bounded versions of D and I with bounded arities.
} 


\subsection{Relativised determinacy operators}

The determinacy of a formula by a set of formulae can be relativised to a set of possible worlds. Let $\llbracket \theta \rrbracket_{W}$ denote the set $\{w \in W \mid W, w \models \theta\}$ and define the relativised determinacy operator $\mathrm{D}^{\theta}\left(\varphi_{1}, \ldots, \varphi_{k} ; \psi\right)$ such that $W, w \models \mathrm{D}^{\theta}\left(\varphi_{1}, \ldots, \varphi_{k} ; \psi\right)$ iff for all $u, v \in \llbracket \theta \rrbracket_{W}$, if the equivalence

$$
W, u \models \varphi_{i} \Leftrightarrow W, v \models \varphi_{i}
$$

holds for each $i \leq k$, then we have

$$
W, u \models \psi \Leftrightarrow W, v \models \psi .
$$

In particular, we define $\mathrm{C}^{\theta} \varphi=\mathrm{D}^{\theta}(\emptyset, \varphi)$. Using $[\mathrm{u}]$, we notice that

$$
\mathrm{C}^{\theta} \varphi \equiv[\mathrm{u}](\theta \rightarrow \varphi) \vee[\mathrm{u}](\theta \rightarrow \neg \varphi) .
$$

We also notice that $\mathrm{D}(\theta, \varphi)$ is definable in terms of $\mathrm{C}^{\theta}$ as follows.

$$
\mathrm{D}(\theta, \varphi) \equiv \mathrm{C}^{\theta} \varphi \wedge \mathrm{C}^{\neg \theta} \varphi
$$

Furthermore, $\mathrm{D}^{\theta}$ is inductively definable in terms of $\mathrm{C}^{\theta}$ as follows.

1. $\mathrm{D}^{\theta}(\emptyset, \varphi)=\mathrm{C}^{\theta} \varphi$

2. $\mathrm{D}^{\theta}\left(\varphi_{1}, \ldots, \varphi_{k+1}, \psi\right)$

$$
\equiv \mathrm{D}^{\theta \wedge \varphi_{k+1}}\left(\varphi_{1}, \ldots, \varphi_{k}, \psi\right) \wedge \mathrm{D}^{\theta \wedge \neg \varphi_{k+1}}\left(\varphi_{1}, \ldots, \varphi_{k}, \psi\right)
$$

We provide a simple example illustrating the use of relativised determinacy in natural language. Consider a scenario with the following propositions.

- There are road blocks, denoted by $p$.

- It is rush hour, denoted by $q$.

- John will be on time, denoted by $r$.

Assume the set of possible worlds in the scenario is $W=\left\{w_{1}, w_{2}, w_{3}, w_{4}, w_{5}\right\}$, where

- $w_{1}=\{(p, 0),(q, 0),(r, 1)\}$,

- $w_{2}=\{(p, 0),(q, 1),(r, 1)\}$,

- $w_{3}=\{(p, 0),(q, 1),(r, 0)\}$,

- $w_{4}=\{(p, 1),(q, 0),(r, 1)\}$,

- $w_{5}=\{(p, 1),(q, 1),(r, 0)\}$. 
Consider the following claim.

If there are road blocks, then, whether it is rush hour determines whether John will be on time.

Perhaps the most natural interpretation for this sentence is given by $\mathrm{D}^{p}(q, r)$, which is true in each world of the model $W=\left\{w_{1}, \ldots, w_{5}\right\}$, and thus we have $W \models \mathrm{D}^{p}(q, r)$. Interestingly, however, there is at least one sensible alternative interpretation, $p \rightarrow \mathrm{D}(q, r)$, which is true in the worlds $w_{1}, w_{2}, w_{3}$, and false in $w_{4}, w_{5}$, whence we have $W \not \models p \rightarrow \mathrm{D}(q, r)$.

\subsection{Determinacy and independence in the general modal setting}

As already mentioned in the introduction section, the determinacy operator D can be naturally generalised to the general modal setting. Given a Kripke model $M=(W, R, V)$ and a possible world $w \in W$, we define the semantics of $\mathrm{D}$ as follows: $M, w \models \mathrm{D}\left(\varphi, \ldots, \varphi_{k} ; \psi\right)$ iff for all $u, v \in W$ such that $w R u$ and $w R v$, if the equivalence $M, u \models \varphi_{i} \Leftrightarrow M, v \models \varphi_{i}$ holds for each $i \leq k$, then $M, u \models \psi \Leftrightarrow M, v \models \psi$.

It is easy to see that in a propositional language extended with both $D$ and the standard box modality $\square$, we have the equivalence

$$
\mathrm{D}\left(\varphi_{1}, \ldots, \varphi_{k} ; \psi\right) \equiv \bigvee_{\chi \in D N F\left(\varphi_{1}, \ldots, \varphi_{k}\right)} \square(\chi \leftrightarrow \psi),
$$

i.e., for all Kripke models $M$ and points $w$ in the domain of $M$, the pointed model $M, w$ satisfies either both or neither of the above formulae. On the other hand, on all models with a reflexive accessibility relation (but not in general), the box modality $\square$ is definable in terms of $D$ by $\square \varphi:=\varphi \wedge C \varphi$, where $C \varphi$ of course denotes $\mathrm{D}(\emptyset ; \varphi)$.

Studying D over different classes of Kripke models provides an interesting research direction. In fact, the recent study [Fan16] has already taken up that direction.

\subsection{Logical determinacy and consequence}

It is possible to extend the scope of $D$ to cover arbitrary sets of formulae as follows. Let $\Gamma$ denote a possibly infinite set of formulae of $\mathcal{L}_{\mathrm{D}}$. Define $W, w \models \mathrm{D}(\Gamma, \psi)$ if for all assignments $u, v \in W$ it holds that if the equivalence $W, u \models \varphi \Leftrightarrow W, v \models \varphi$ holds for all $\varphi \in \Gamma$, then $W, u \models \psi \Leftrightarrow W, v \models \psi$.

The determinacy operator D now parallels in a natural way Tarski's notion of a logical consequence operator $\mathcal{C}$ defined so that $\mathcal{C}(\Gamma, \psi)$ holds if and only if $W, w \models \psi$ for every state description model $W$ and assignment $w$ such that $W, w \models \varphi$ for all $\varphi \in \Gamma$. The parallel is in the sense that D satisfies the same defining properties (Reflexivity, Monotonicity and Cut) which Tarski postulated for $\mathcal{C}$ (while D has some interesting extra properties that $\mathcal{C}$ lacks). Indeed, this is not accidental, because $\mathrm{D}$ and $\mathcal{C}$ bear technically similar ideas: $\mathcal{C}$ preserves truth, whereas $\mathrm{D}$ preserves invariance of truth values. For a further discussion of this and related 
issues, see [Hum92, Hum93, Hum98], as well as [Cia16a, Cia16b] for an argument presenting dependence as a case of logical consequence applied to questions instead of propositions. Thus, an interesting research direction extending the present work involves relating determinacy and logical consequence in the more general modal setting outlined here.

\subsection{Determinacy operators and conditional knowledge}

The determinacy operator $D$ has a natural epistemic reading: the determinacy formula $\mathrm{D}\left(\varphi_{1}, \ldots, \varphi_{k}, \psi\right)$ can be interpreted to mean that an agent knows the truth value of $\psi$ relative to the truth values of the formulae $\varphi_{1}, \ldots, \varphi_{k}$ in the sense that the agent can always deduce the truth value of $\psi$ if she learns the truth values of $\varphi_{1}, \ldots, \varphi_{k}$. This interpretation of D leads to yet another open research direction. It is worth noting that a uniform analysis of various kinds of knowledge, including knowledge of questions and knowledge of dependencies of this kind has already been given in the work CR15] on inquisitive epistemic logic (IEL). Indeed, conditional knowledge can be regarded as knowledge obtained by answering questions: the formula $\mathrm{D}(\varphi ; \psi)$ with the meaning "agent $a$ knows whether $\psi$ holds conditionally on the knowledge whether $\varphi$ holds" can be expressed in IEL as $K_{a}(? \varphi \rightarrow ? \psi)$.

Acknowledgements. Valentin Goranko was partly supported by a research grant 2015-04388 of the Swedish Research Council. Antti Kuusisto was supported by Jenny and Antti Wihuri Foundation and the ERC grant 647289 (CODA). The results of this paper were presented at the Dagstuhl seminar Logics for Dependence and Independence in June 2015, where an earlier manuscript of the present paper was circulated; we thank the participants for their interest. After submitting the arXiv preprint GK16, we have also been in correspondence with Jie Fan and Lloyd Humberstone. We thank them for their comments and discussion. In particular, Humberstone subsequently commented in depth some aspects of our work in [Hum17] and Jie Fan produced the manuscript [Fan16].

\section{References}

[Alo16] Maria Aloni. Disjunction. In Edvard N. Zalta, editor, The Stanford Encyclopedia of Philosophy. Stanford University, Stanford, 2016.

[Arm74] William Armstrong. Dependency structures of database relationships. In IFIP' ' 4 , pages 580-583, 1974.

[Cia09] Ivano Ciardelli. Inquisitive semantics and intermediate logics. Master's thesis, Institute of Logic, Language and Computation, University of Amsterdam, 2009.

[Cia16a] Ivano Ciardelli. Dependency as question entailment. In Jouko Väänänen Samson Abramsky, Juha Kontinen and Heribert Vollmer, editors, Dependence Logic: theory and applications, pages 129-181. Springer International Publishing, Switzerland, 2016. 
[Cia16b] Ivano Ciardelli. Questions in Logic. PhD thesis, Institute of Logic, Language and Computation, University of Amsterdam, 2016.

[CR11] Ivano Ciardelli and Floris Roelofsen. Inquisitive logic. Journal of Philosophical Logic, 40(1):55-94, 2011.

[CR15] Ivano Ciardelli and Floris Roelofsen. Inquisitive dynamic epistemic logic. Synthese, 192(6):1643-1687, 2015.

[Dem97] Stéphane Demri. A completeness proof for a logic with an alternative necessity operator. Studia Logica, 58(1):99-112, 1997.

[Fan16] Jie Fan. A modal logic of supervenience. Manuscript, arXiv:1611.04740v1, 2016.

[FWvD15] Jie Fan, Yanjing Wang, and Hans van Ditmarsch. Contingency and knowing whether. Review of Symbolic Logic, 8(1):75 -107, 2015.

[Gal12] Pietro Galliani. Inclusion and exclusion dependencies in team semantics - on some logics of imperfect information. Annals of Pure and Applied Logic, 163(1):68-84, 2012.

[Gal13] Pietro Galliani. Epistemic operators in dependence logic. Studia Logica, 101(2):367-397, 2013.

[GK16] Valentin Goranko and Antti Kuusisto. Logics for propositional determinacy and independence. Manuscript, arXiv:1609.07398, 2016.

[GP92] Valentin Goranko and Solomon Passy. Using the universal modality: Gains and questions. Journal of Logic and Computation, 2(1):5-30, 1992.

[Gre39] Kurt Grelling. A logical theory of dependence. In Proceedings of the 5th International Congress for the Unity of Science, 1939.

[GV13] Erich Grädel and Jouko Väänänen. Dependence and independence. Studia Logica, 101(2):399-410, 2013.

[GV14] Pietro Galliani and Jouko Väänänen. On dependence logic. In A. Baltag and S. Smets, editors, Johan F. A. K. van Benthem on Logical and Informational Dynamics, pages 101-119. Springer, 2014.

[Hin96] Jaakko Hintikka. The Principles of Mathematics Revisited. Cambridge UP, 1996.

[HKMV15] Lauri Hella, Antti Kuusisto, Arne Meier, and Heribert Vollmer. Modal inclusion logic: Being lax is simpler than being strict. In MFCS 2015, Proceedings, Part I, pages 281-292, 2015. 
[HKVV15] Miika Hannula, Juha Kontinen, Jonni Virtema, and Heribert Vollmer. Complexity of propositional independence and inclusion logic. In MFCS 2015, Proceedings, Part I, pages 269-280, 2015.

[HLSV14] Lauri Hella, Kerkko Luosto, Katsuhiko Sano, and Jonni Virtema. The expressive power of modal dependence logic. In R. Goré, B. Kooi, and A. Kurucz, editors, Advances in Modal Logic 10, pages 294-312. College Publications, 2014.

[Hod97] Wilfrid Hodges. Compositional semantics for a language of imperfect information. Logic Journal of the IGPL, 5(4):539-563, 1997.

[HS89] Jaakko Hintikka and Gabriel Sandu. Informational independence as a semantical phenomenon. In J. E. Fenstad, I. T. Frolov, and R. Hilpinen, editors, Logic, Methodology and Philosophy of Science, volume 8, pages 571-589. Elsevier, Amsterdam, 1989.

[Hum92] Lloyd Humberstone. Some structural and logical aspects of the notion of supervenience. Logique et Analyse, 35:101-137, 1992.

[Hum93] Lloyd Humberstone. Functional dependencies, supervenience, and consequence relations. Journal of Logic, Language and Information, 2(4):309-336, 1993.

[Hum95] Lloyd Humberstone. The logic of non-contingency. Notre Dame Journal of Formal Logic, 36(2):214-229, 1995.

[Hum98] Lloyd Humberstone. Note on supervenience and definability. Notre Dame Journal of Formal Logic, 39(2):243-252, 1998.

[Hum02] Lloyd Humberstone. The modal logic of agreement and noncontingency. Notre Dame Journal of Formal Logic, 43(2):95-127, 2002.

[Hum17] Lloyd Humberstone. Supervenience, dependence, disjunction. Manuscript, June 2017.

[Jan97] Theo Janssen. An overview of compositional translations. In Compositionality: The Significant Difference, International Symposium, COMPOS'97, Bad Malente, Germany, September 8-12, 1997. Revised Lectures, pages 327-349, 1997.

[KMSV14] Juha Kontinen, Julian-Steffen Müller, Henning Schnoor, and Heribert Vollmer. Modal independence logic. In Advances in Modal Logic 10, pages 353-372, 2014.

[KMSV15] Juha Kontinen, Julian-Steffen Müller, Henning Schnoor, and Heribert Vollmer. A van benthem theorem for modal team semantics. In CSL 2015, Proceedings, pages 277-291, 2015. 
[Kon13] Juha Kontinen. Dependence logic: A survey of some recent work. Philosophy Compass, 8(10):950-963, 2013.

[Kuu14] Antti Kuusisto. A double team semantics for generalized quantifiers. CoRR, abs/1310.3032v5, 2014.

[Kuu15] Antti Kuusisto. A double team semantics for generalized quantifiers. CoRR, abs/1310.3032v8, 2015.

[LV13] Peter Lohmann and Heribert Vollmer. Complexity results for modal dependence logic. Studia Logica, 101(2):343-366, 2013.

[MB14] Brian McLaughlin and Karen Bennett. Supervenience. In Edward N. Zalta, editor, The Stanford Encyclopedia of Philosophy. Stanford, 2014.

[MR66] Hugh Montgomery and Richard Routley. Contingency and noncontingency bases for normal modal logics. Logique et Analyse, 9(35):318-328, 1966.

[Piz07] Claudio Pizzi. Necessity and relative contingency. Studia Logica, 85(3):395-410, 2007.

[Piz13] Claudio Pizzi. Relative contingency and bimodality. Logica Universalis, 7(1):113-123, 2013.

[Roe13] Floris Roelofsen. Algebraic foundations for the semantic treatment of inquisitive content. Synthese, 190(1):79-102, 2013.

[SvE88] Barry Smith and Christina von Ehrenfels. Foundations of Gestalt Theory. Philosophia resources library. Philosophia Verlag, 1988.

[Vää07] Jouko Väänänen. Dependence Logic - A New Approach to Independence Friendly Logic, volume 70 of London Mathematical Society student texts. Cambridge University Press, 2007.

[Vää08] Jouko Väänänen. Modal dependence logic. In K. R. Apt and R. van Rooij, editors, New Perspectives on Games and Interaction, pages 237254. Amsterdam University Press, 2008.

[Yan14] Fan Yang. Extensions and Variants of Dependence Logic. PhD thesis, University of Helsinki, 2014.

[Yan16] Fan Yang. Uniform definability in propositional dependence logic. arXiv:1501.00155, 2016.

[YV16] Fan Yang and Jouko Väänänen. Propositional logics of dependence. Annals of Pure and Applied Logic, 167(7):557-589, 2016. 